Rapporto n. 222

Interval Estimation for the Sortino Ratio and the Omega Ratio

Lucio De Capitani

dicembre 2011

Dipartimento di Metodi Quantitativi per le Scienze Economiche ed Aziendali Università degli Studi di Milano Bicocca

Via Bicocca degli Arcimboldi , 8 - 20126 Milano - Italia

$\mathrm{Tel}+39 / 02 / 64483103-\mathrm{Fax}+39 / 02 / 64483105$

Segreteria di redazione: Andrea Bertolini 


\title{
Interval estimation for the Sortino Ratio and the Omega Ratio
}

\author{
Lucio De Capitani*
}

\begin{abstract}
In this paper asymptotic confidence intervals for the Sortino and Omega Ratio are proposed and analyzed. First the confidence intervals are derived under the strong assumption of temporal independence and identical distribution of the returns. Later they are obtained assuming that the process followed by returns is strictly stationary and $\alpha$-mixing of a certain size. In order to evaluate the minimum sample size for a good coverage accuracy of the asymptotic confidence intervals, a simulation study is performed. It is obtained that the minimum sample sizes are very high, especially under the more realistic assumption of not-i.i.d. returns.
\end{abstract}

keywords: financial performance ratio, dependent central limit theorem, strong mixing condition, coverage probability, GARCH model.

\section{Introduction}

The performance of financial assets is usually measured by means of the ratio of a reward measure and a risk measure. The Sharpe Ratio (Sharpe , 1964, 1966, 1994) is the most important example of this kind of measure and it can be considered the "father" of a plethora of ratios such as the Sortino Ratio (Sortino and Van der Meer , 1991; Sortino and Price , 1994), the MAD Ratio (Konno and Yamazaki , 1991), the MD Ratio (Shalit and Yitzhaki , 1984), the MiniMax Ratio, the Stable Ratio (see Farinelli et al , 2008, and the references therein) and the recently introduced Omega function (Keating and Shadwick , 2002). All the aforementioned indicators depend on some unknown features of the returns distribution. As a consequence the performance ratios associated to a particular financial asset are unknown and they are usually estimated starting from an observed time series of returns. In this situation, a confidence interval for the value of the ratios is very useful since its length incorporates a precious information about the variability of the point estimator and, then, it sheds light on the uncertainty of the estimates. Confidence intervals for the Sharpe Ratio have been developed in Lo (2002), Opdike (2007), and De Capitani (2010). The confidence interval for the MAD ratio and for the MD ratio are obtained in De Capitani and Zenga

*Department of Quantitative Methods for Business and Economic Sciences. - University of MilanoBicocca. - Via Bicocca degli Arcimboldi, 8, 20126 Milano, Italy. Tel.: +39 $0264483186 . \quad$ Fax: +39 0264483105. email: lucio.decapitani1@unimib.it 
(2011) under the assumption of i.i.d. returns. In this paper we derive the confidence intervals for the Sortino Ratio and for Omega function. In more details, the paper is organized as follows. In Section 2 we recall the definitions of the Sortino Ratio and the Omega function and we introduce their natural estimators. In Section 3 we recall the general central limit theorem for dependent observations due to Ibragimov (see Ibragimov , 1962) and we prove a corollary to the aforementioned theorem which is very useful to derive the large sample distribution of the Sortino Ratio and Omega Ratio estimators. In Section 4 we obtain the asymptotic confidence interval for the Sortino Ratio under the assumption of i.i.d. returns and, later, in the more general scenario in which returns follow a strictly stationary and $\alpha$-mixing process. Section 5 concerns the asymptotic confidence intervals for the Omega function. In Section 6 we describe the simulation study performed in order to asses the coverage accuracy of the aforementioned confidence intervals. In Section 7 we discuss the simulation results while Section 8 is devoted to the conclusions.

\section{The Sortino Ratio and the Omega Ratio}

The Sortino Ratio and the Omega function can be considered descendant of the Sharpe Ratio $(\psi)$. Then, we think it is useful to briefly recall the definition and the meaning of $\psi$. Let $X$ be the random variable describing the log-returns of a risky financial activity and let $\xi$ be the (log) risk-free rate of return. Let $f_{X}$ and $F_{X}$ be the density and distribution functions of $X$, respectively. Finally, denote by $\mu_{X}$ and $\sigma_{X}$ the expected value and the standard deviation of $X$. The Sharpe Ratio is defined as

$$
\psi=\frac{\mu_{X}-\xi}{\sigma_{X}}
$$

The value of $\psi$ can be interpreted as the expected excess return per unit of risk where the risk is measured by the standard deviation of the returns. The Sortino Ratio $(v)$ was proposed by Sortino and Van der Meer (1991) as an alternative to the Sharpe ratio. In order to define $v$, let $k$ be a target return which can be intended as the (individual) threshold beyond which an investment starts to produce income for a particular investor. In what follows we always assume that $k$ is a known constant. The Sortino Ratio is defined as:

$$
v=\frac{\mu_{X}-k}{\sigma_{X}^{-}(k)} \quad \text { where } \quad \sigma_{X}^{-}(k)=\left(\int_{-\infty}^{k}(x-k)^{2} f_{X}(x) d x\right)^{\frac{1}{2}} .
$$

The quantity $\sigma_{X}^{-}(k)$ is usually called downside deviation and it can be considered the main element of innovation with respect to the Sharpe Ratio. In more detail, a criticism commonly raised against the Sharpe Ratio is that the variability index $\sigma_{X}$, employed by it as risk measure, gives equal weight to the variability of the returns greater than $\mu_{X}$ (which can be interpreted as "good" variability) and to the variability of returns lower than the $\mu_{X}$ (interpretable as "worst" variability). The use of the downside deviation $\sigma_{X}^{-}(k)$ tries to overcome this criticism since it takes into account only the returns lower than the threshold $k$. In analogy with $\psi, v$ can be interpreted as the expected excess return per unit of risk where the risk is measured by the downside deviation and the excess return is defined with respect to the target $k$. 
The performance measure known as Omega function $(\Omega)$ has been recently introduced by Keating and Shadwick (2002). This measure can be defined as follows:

$$
\begin{aligned}
\Omega & =\frac{\int_{k}^{\infty}\left(1-F_{X}(x)\right) d x}{\int_{-\infty}^{k} F_{X}(x) d x} \\
& =1+\frac{\mu_{X}-k}{\delta_{X}^{-}(k)}
\end{aligned}
$$

where

$$
\delta_{X}^{-}(k)=\int_{-\infty}^{k}|x-k| f_{X}(x) d x
$$

can be called downside mean absolute deviation. Expression (1) is the definition of the Omega function as proposed by Keating and Shadwick (2002) while expression (2) can be easily obtained observing that $\left(\mu_{X}-k\right)=\int_{k}^{\infty}\left(1-F_{X}(x)\right) d x-\int_{-\infty}^{k} F_{X}(x) d x$. From expression (2) it can be seen that the quantity $(\Omega-1)$ has a structure very similar to that of the Sortino Ratio. In particular, the quantity

$$
\omega=\Omega-1=\frac{\mu_{X}-k}{\delta_{X}^{-}(k)}
$$

can be interpreted as the "downside counterpart" of the MAD Ratio which is given by (see, for example, De Capitani and Zenga , 2011):

$$
\psi_{\delta}=\frac{\mu_{X}-\xi}{\delta_{X}} \quad \text { where } \quad \delta_{X}=\int_{-\infty}^{\infty}|x-\mu| f_{X}(x) d x
$$

Roughly speaking, we can say that "the Sortino Ratio is related to the Sharpe Ratio such as $\omega$ is related to the MAD Ratio". For this reason, in the following, we will prefer to study the features of $\omega$ instead those of $\Omega$ and we will call $\omega$ the Omega Ratio. For completeness, we recall that $\omega$ can be interpreted as the expected excess return per unit of risk where the risk is measured by the downside mean absolute deviation and the excess return is defined with respect to the target $k$.

The Sortino and Omega Ratio of a particular financial asset can be estimated starting from a time series of log-returns. In particular, let $X_{1}, X_{2}, \ldots, X_{n}$ be a time series of logreturns and consider the following estimators:

$$
\begin{aligned}
& \bar{X}=\frac{1}{n} \sum_{i=1}^{n} X_{i} \\
& \hat{\sigma}_{X}^{-}(k)=\left(\frac{1}{n} \sum_{i=1}^{n}\left(X_{i}-k\right)^{2} \mathbb{I}_{k}\left(X_{i}\right)\right)^{\frac{1}{2}} \quad \text { where } \mathbb{I}_{k}\left(X_{i}\right)=\left\{\begin{array}{ll}
1 & X_{i} \leq k \\
0 & \text { otherwise }
\end{array} ;\right. \\
& \hat{\delta}_{X}^{-}(k)=\frac{1}{n} \sum_{i=1}^{n}\left(k-X_{i}\right) \mathbb{I}_{k}\left(X_{i}\right) .
\end{aligned}
$$


The natural estimators of $v$ and $\omega$ can, then, be introduced:

$$
\begin{aligned}
& \widehat{\Upsilon}=\frac{\bar{X}-k}{\hat{\sigma}_{X}^{-}(k)} ; \\
& \widehat{\Omega}=\frac{\bar{X}-k}{\hat{\delta}_{X}^{-}(k)} .
\end{aligned}
$$

The following sections aim to derive the asymptotic distribution of the estimators (3) and (4). This objective can be reached in an easier way focusing directly on the excess returns $Y_{i}=X_{i}-k$ with $i=1, \ldots, n$. Indeed, with this change of notation, all the expressions required to describe the large sample distributions of (3) and (4) result more elegant and interpretable. Moreover, in order to further ease the notation, we will drop the subscript $Y$ and the indication of the target return $k$ from the symbols denoting the moments or the relevant quantity relative to the excess return random variable $Y=X-k$. For example, the expectation of $Y$ will be denoted simply by $\mu$, the quantity $\delta_{X}^{-}(k)=\delta_{Y}^{-}(0)$ will be denoted by $\delta^{-}$, and so on. Finally, note that using the simplified notation we have: $\widehat{\Upsilon}=\frac{\bar{Y}}{\hat{\sigma}^{-}}$and $\widehat{\Omega}=\frac{\bar{Y}}{\hat{\delta}^{-}}$.

\section{Recalling the Ibragimov Central Limit Theorem for stationary $\alpha$-mixing processes}

If the returns are assumed i.i.d., the asymptotic distributions of $\widehat{\Upsilon}$ and $\widehat{\Omega}$ can be obtained starting from the classical and well known multivariate central limit theorem (see, e.g., Serfling , 1980). On the contrary, if the independence assumption is relaxed, a more general Central Limit Theorem (CLT) for dependent observations is necessary. In literature there are several CLTs of this kind, all based on quite different regularity conditions. Even if these limit theorems was proposed several years ago (from 1960 to 1980), nowadays their knowledge is not as widespread as that of the limit theorems for i.i.d. observations. For this reason, in this section we briefly recall the CLT for stationary $\alpha$-mixing (strong mixing) processes proposed by Ibragimov (1962). This theorem is the fundamental results we use to derive the asymptotic distribution of $\widehat{\Upsilon}$ and $\widehat{\Omega}$ when returns are dependent.

Let $(\Omega, \mathcal{F}, P)$ be a probability space and let $\mathcal{G}$ and $\mathcal{H}$ be two $\sigma$-subfield of $\mathcal{F}$. The $\sigma$ subfields $\mathcal{G}$ and $\mathcal{H}$ are independent if for all $G \in \mathcal{G}$ and $H \in \mathcal{H}$ it turns out that $P(G \cap H)=$ $P(G) P(H)$. As a measure of dependence between $\mathcal{G}$ and $\mathcal{H}$, the strong mixing coefficient is usually adopted:

$$
\alpha(\mathcal{G}, \mathcal{H})=\sup _{G \in \mathcal{G} ; H \in \mathcal{H}}|P(G \cap H)-P(G) P(H)| .
$$

Now, let $\left\{X_{t}\right\}_{t \in \mathbb{N}}=\left\{X_{1}, X_{2}, \ldots\right\}$ be a one-sided strictly stationary and ergodic (for the definition of ergodicity, we refer the interested reader to Karlin and Taylor , 1975) stochastic process. As it is well known (see Billingsley , 1999, Appendix M22), the process $\left\{X_{t}\right\}_{t \in \mathbb{N}}$ can be extended to a stationary and ergodic two-sided process $\left\{X_{t}\right\}_{t \in \mathbb{Z}}=\left\{\ldots, X_{-1}, X_{0}, X_{1}, \ldots\right\}$. The one-sided and two-sided processes have the same finite-dimensional distributions. For this reason they can be considered equivalent for our purposes since "all the convergence 
in distributions results" depend only on the finite dimensional distributions. Then, all the results described in this section hold both for $\left\{X_{t}\right\}_{t \in \mathbb{N}}$ and for $\left\{X_{t}\right\}_{t \in \mathbb{Z}}$. Usually, this results are derived for the two-sided process $\left\{X_{t}\right\}_{t \in \mathbb{Z}}$ and we follow this custom even if, in our application, it is usual to work with the one-sided process.

Now, the two-sided stationary sequences $\left\{X_{t}\right\}_{t \in \mathbb{Z}}$ is said to be $\alpha$-mixing (or strong mixing) if $\lim _{m \rightarrow \infty} \alpha_{m}=0$ where

$$
\alpha_{m}=\alpha\left(\mathcal{F}_{-\infty}^{t}, \mathcal{F}_{t+m}^{\infty}\right)
$$

$\mathcal{F}_{-\infty}^{t}=\sigma\left(\ldots, X_{t-1}, X_{t}\right)$, and $\mathcal{F}_{t+m}^{\infty}=\sigma\left(X_{t+m}, X_{t+m+1}, \ldots\right)$. Obviously, the slower the convergence to 0 of the sequence $\left\{\alpha_{m}\right\}$, the stronger the persistence of the time dependence. The evaluation of the rate of convergence of $\left\{\alpha_{m}\right\}$ is fundamental for the development of a central limit theorem and it is usually evaluated introducing the notion of size of a $\alpha$-mixing process. In more detail, a stationary process is $\alpha$-mixing of size $-\varphi$ if $\alpha_{m}=O\left(m^{-\varphi^{*}}\right)$ for some $\varphi^{*}>\varphi$. In this case it turns out that

$$
\sum_{m=1}^{\infty} \alpha_{m}^{1 / \varphi}<\infty
$$

It is well known that a stationary $\alpha$-mixing process is ergodic and, moreover, the strong mixing condition is preserved by measurable transformations, as stated below (see Davidson , 1994, p. 210).

Proposition 1 Let $\left\{X_{t}\right\}_{t \in \mathbb{Z}}$ be stationary and $\alpha$-mixing of size $-\varphi$ and let $Y_{t}=g\left(X_{t}\right)$ be a measurable function. Then the process $\left\{Y_{t}\right\}_{t \in \mathbb{Z}}$ is stationary and $\alpha$-mixing of size $-\varphi$.

Now, we are ready to recall the following central limit theorem due to Ibragimov (1962) (see, for istance, Hall and Heyde, 1980, p. 132).

Theorem 1 Let $0<\delta<\infty$ be fixed and let $\left\{X_{t}\right\}_{t \in \mathbb{Z}}$ be stationary and $\alpha$-mixing of size $-\frac{2+\delta}{\delta}$ with $E\left[X_{t}\right]=\mu$ and $E\left[\left|X_{t}\right|^{2+\delta}\right] \leq \infty$. Then

$$
\sum_{i=1}^{\infty} \alpha_{m}^{\delta /(2+\delta)}<\infty \quad \text { and } \quad \lim _{n \rightarrow \infty} n E\left[(\bar{X}-\mu)^{2}\right]=V
$$

Moreover, if $V>0$, then $\sqrt{n}(\bar{X}-\mu) \stackrel{d}{\rightarrow} \mathcal{N}(0, V)$.

Now, in the following it will be used the corollary to Theorem 1 stated below.

Corollary 1 Let $\left\{X_{t}\right\}_{t \in \mathbb{Z}}$ be stationary and $\alpha$-mixing of size $-\frac{2+\delta}{\delta}$ with $0<\delta<\infty$. Let $Y_{t}=g\left(X_{t}\right)$ be a measurable function and assume that

$$
E\left[X_{t}\right]=\mu, \quad E\left[Y_{t}\right]=\mu_{Y}, \quad \text { and } \max \left\{E\left[\left|X_{t}\right|^{2+\delta}\right] ; E\left[\left|g\left(X_{t}\right)\right|^{2+\delta}\right]\right\}<\infty .
$$

Let $\mathbf{S}_{n}$ denote the variance-covariance matrix of the random vector $\sqrt{n}\left[\begin{array}{c}\bar{X}-\mu \\ \bar{Y}-\mu_{Y}\end{array}\right]$. Then, $\lim _{n \rightarrow \infty} \mathbf{S}_{n}=\mathbf{S}$ is finite and if it is positive-definite then

$$
\sqrt{n}\left[\begin{array}{c}
\bar{X}-\mu \\
\bar{Y}-\mu_{Y}
\end{array}\right] \stackrel{d}{\rightarrow} \mathcal{N}(\mathbf{0}, \mathbf{S})
$$




\section{Proof}

Let $\mathbf{S}_{i j}$ denote the element in row $i$ and column $j$ of $\mathbf{S}$. Note that the positive definiteness of $\mathbf{S}$ assures that $S_{i i}>0(i=1,2)$. Moreover, Theorem 1 assures that $S_{i i}<\infty(i=$ $1,2)$ and, from the Cauchy-Schwarz inequality, we have $\mathbf{S}_{12}=\mathbf{S}_{21}<\infty$. In addition, the positive definiteness of $\mathbf{S}$ assures that $\lambda^{\prime} \mathbf{S} \lambda>0$ for all vectors $\lambda=\left(\lambda_{1}, \lambda_{2}\right)^{\prime} \neq(0,0)$. Then, from Theorem 1 it directly follows that $\sqrt{n}(\bar{X}-\mu) \stackrel{d}{\rightarrow} \mathcal{N}\left(0, \mathbf{S}_{11}\right)$. Analogously, thanks to Proposition 1 we have that $\sqrt{n}\left(\bar{Y}-\mu_{Y}\right) \stackrel{d}{\rightarrow} \mathcal{N}\left(0, \mathbf{S}_{\mathbf{2 2}}\right)$ and $\sqrt{n}\left(\lambda_{1} \bar{X}+\lambda_{2} \bar{Y}-\lambda_{1} \mu-\lambda_{2} \mu_{Y}\right) \stackrel{d}{\rightarrow}$ $\mathcal{N}\left(0, \lambda^{\prime} \mathbf{S} \lambda\right)$ for all $\lambda \neq \mathbf{0}$. Now, the Corollary follows from the Cramer-Wold device.

Finally, we state the following proposition, which will be very useful in the next sections.

Proposition 2 Let $\left\{X_{t}\right\}_{t \in \mathbb{Z}}$ be stationary and $\alpha$-mixing of size $-\frac{2+\delta}{\delta}$ with $0<\delta<\infty$. If $E\left[\left|X_{t}\right|^{2+\delta}\right]<\infty$ then

$$
\sum_{m=1}^{\infty}\left|\operatorname{Cov}\left(X_{1}, X_{1+m}\right)\right|<\infty .
$$

Proof

The proposition follows form Corollary A.2 on page 278 of Hall and Heyde (1980). In more detail, from this corollary we have that:

$$
\left|\operatorname{Cov}\left(X_{1}, X_{1+m}\right)\right| \leq 8 E\left[\left|X_{t}\right|^{2+\delta}\right]^{\frac{2}{2+\delta}} \alpha_{m}^{\frac{\delta}{2+\delta}} .
$$

Now the proposition follows from $\sum_{i=1}^{\infty} \alpha_{m}^{\frac{\delta}{2+\delta}}<\infty$ which is assured by the fact that $\left\{X_{t}\right\}_{t \in \mathbb{Z}}$ is stationary and $\alpha$-mixing of size $-\frac{2+\delta}{\delta}$.

\section{Asymptotic distributions of $\widehat{\Upsilon}$ and confidence inter- val for $v$}

\subsection{The case of i.i.d returns}

First, let us assume that the sample excess returns $Y_{1}, Y_{2} \ldots, Y_{n}$ are i.i.d.. Obviously, the latter is a strong assumption and it is clearly confuted by the empirical evidence. However we think it is important to analyze first this particular case since it furnishes a useful starting point for the more complex analysis we will make in the following. As in Lo (2002) and De Capitani and Zenga (2011), the asymptotic distribution of $\widehat{\Upsilon}$ will be obtained applying the delta method to the large sample distribution of the random vector

$$
\left[\begin{array}{c}
\bar{Y} \\
\left(\hat{\sigma}^{-}\right)^{2}
\end{array}\right]=\frac{1}{n} \sum_{i=1}^{n}\left[\begin{array}{c}
Y_{i} \\
Y_{i}^{2} \mathbb{I}_{0}\left(Y_{i}\right)
\end{array}\right] .
$$

In order to obtain the asymptotic distribution of (5) we derive its variance-covariance matrix. First, we recall that

$$
E[\bar{Y}]=\mu \quad \text { and } \quad \operatorname{Var}(\bar{Y})=\frac{\sigma^{2}}{n} .
$$


Moreover, we have:

$$
\begin{aligned}
E\left[\left(\hat{\sigma}^{-}\right)^{2}\right] & =E\left[\frac{1}{n} \sum_{i=1}^{n} Y_{i}^{2} \mathbb{I}_{0}\left(Y_{i}\right)\right] \\
& =E\left[Y_{1}^{2} \mathbb{I}_{0}\left(Y_{1}\right)\right] \\
& =\int_{-\infty}^{0} y^{2} f_{Y}(y) d y \\
& =\mu_{2}^{-}
\end{aligned}
$$

Analogously, since the $Y_{i}^{\prime}$ 's are assumed independent and identically distributed, we obtain:

$$
\begin{aligned}
\operatorname{Var}\left[\left(\hat{\sigma}^{-}\right)^{2}\right] & =\operatorname{Var}\left(\frac{1}{n} \sum_{i=1}^{n} Y_{i}^{2} \mathbb{I}_{0}\left(Y_{i}\right)\right) \\
& =\frac{\operatorname{Var}\left(Y_{1}^{2} \mathbb{I}_{0}\left(Y_{1}\right)\right)}{n} \\
& =\frac{1}{n}\left[\int_{-\infty}^{0} y^{4} f_{Y}(y) d y-\left(\mu_{2}^{-}\right)^{2}\right] \\
& =\frac{\mu_{4}^{-}-\left(\mu_{2}^{-}\right)^{2}}{n}
\end{aligned}
$$

Finally, the covariance between $\bar{Y}$ and $\left(\hat{\sigma}^{-}\right)^{2}$ is:

$$
\begin{aligned}
\operatorname{Cov}\left[\bar{Y} ;\left(\hat{\sigma}^{-}\right)^{2}\right] & =\frac{1}{n^{2}} \operatorname{Cov}\left[\sum_{i=1}^{n} Y_{i} ; \sum_{i=1}^{n} Y_{i}^{2} \mathbb{I}_{0}\left(Y_{i}\right)\right] \\
& =\frac{1}{n^{2}} E\left[\left(\sum_{i=1}^{n} Y_{i}\right) \cdot\left(\sum_{i=1}^{n} Y_{i}^{2} \mathbb{I}_{0}\left(Y_{i}\right)\right)\right]-\mu \cdot \mu_{2}^{-} \\
& =\frac{1}{n^{2}} \sum_{i=1}^{n} \sum_{j=1}^{n} E\left[Y_{i} \cdot Y_{j}^{2} \mathbb{I}_{0}\left(Y_{j}\right)\right]-\mu \cdot \mu_{2}^{-} \\
& =\frac{1}{n^{2}} n E\left[Y_{1}^{3} \mathbb{I}_{0}\left(Y_{1}\right)\right]+\frac{n^{2}-n}{n^{2}} E\left[Y_{1}\right] E\left[Y_{1}^{2} \mathbb{I}_{0}\left(Y_{1}\right)\right]-\mu \cdot \mu_{2}^{-} \\
& =\frac{\left(\mu_{3}^{-}-\mu \cdot \mu_{2}^{-}\right)}{n} .
\end{aligned}
$$

Now, thank to the multivariate central limit theorem (see, e.g. Serfling, 1980) and to expressions (6), (7), (8), and (9) we can state the following theorem.

Theorem 2 Let $Y$ be a random variable with distribution function $F$ and assume that $E\left[Y^{j}\right]<\infty$ for $j=1,2,3,4$. Let $Y_{1}, \ldots, Y_{n}$ be an i.i.d. sample from $F$. It follows that

$$
\sqrt{n}\left[\begin{array}{c}
\bar{Y}-\mu \\
\left(\hat{\sigma}^{-}\right)^{2}-\mu_{2}^{-}
\end{array}\right] \stackrel{d}{\longrightarrow} \mathbf{B N}(\mathbf{0} ; \boldsymbol{\Sigma})
$$


where BN means "bivariate normal",

$$
\mathbf{0}=\left[\begin{array}{l}
0 \\
0
\end{array}\right], \quad \text { and } \quad \boldsymbol{\Sigma}=\left[\begin{array}{cc}
\sigma^{2} & \mu_{3}^{-}-\mu \cdot \mu_{2}^{-} \\
\mu_{3}^{-}-\mu \cdot \mu_{2}^{-} & \mu_{4}^{-}-\left(\mu_{2}^{-}\right)^{2}
\end{array}\right] .
$$

By applying the delta method, the following corollary is obtained.

Corollary 2 If the assumptions of Theorem 1 are satisfied, it results that

$$
\sqrt{n}(\widehat{\Upsilon}-v) \stackrel{d}{\longrightarrow} \mathcal{N}\left(0 ; V_{v}\right)
$$

where

$$
V_{v}=\frac{\sigma^{2}}{\mu_{2}^{-}}-\left(\frac{\mu_{3}^{-}-\mu \mu_{2}^{-}}{\left(\mu_{2}^{-}\right)^{3 / 2}}\right) v+\left(\frac{\mu_{4}^{-}-\left(\mu_{2}^{-}\right)^{2}}{4\left(\mu_{2}^{-}\right)^{2}}\right) v^{2}
$$

A consistent estimator for the variance $V_{v}$ is

$$
\widehat{V}_{v}=\frac{S^{2}}{\hat{\mu}_{2}^{-}}-\left(\frac{\hat{\mu}_{3}^{-}-\bar{Y}_{\hat{\mu}}^{-}}{\left(\hat{\mu}_{2}^{-}\right)^{3 / 2}}\right) \widehat{\Upsilon}+\left(\frac{\hat{\mu}_{4}^{-}-\left(\hat{\mu}_{2}^{-}\right)^{2}}{4\left(\hat{\mu}_{2}^{-}\right)^{2}}\right) \widehat{\Upsilon}^{2}
$$

where

$$
S^{2}=\frac{1}{(n-1)} \sum_{i=1}^{n}\left(Y_{i}-\bar{Y}\right)^{2}, \quad \hat{\mu}_{j}^{-}=\frac{1}{n} \sum_{i=1}^{n} Y_{i}^{j} \mathbb{I}_{0}\left(Y_{i}\right) \quad \text { with } \quad j=2,3,4 .
$$

Consequently, the following aymptotic $(1-\alpha)$-Confidence Interval (CI) for $v$ can be obtained applying the standard analytical inversion method:

$$
\left(\widehat{\Upsilon}-z_{1-\frac{\alpha}{2}} \sqrt{\frac{\widehat{V}_{v}}{n}} ; \widehat{\Upsilon}+z_{1-\frac{\alpha}{2}} \sqrt{\frac{\widehat{V}_{v}}{n}}\right)
$$

\subsection{The general case of not-i.i.d. returns}

If the sample excess returns are not i.i.d., the large sample distribution of the random vector (5) can be obtained form the Corollary 1 observing that $g: \mathbb{R} \rightarrow \mathbb{R}^{+}, x \mapsto x \mathbb{I}_{0}(x)$ is measurable. However, before specializing Corollary 1 to the analysis of (5) we derive its asymptotic variance-covariance matrix in the case of not-i.i.d. returns.

First, we note that the expectation of $\bar{Y}$ and $\left(\hat{\sigma}_{-}^{2}\right)$ are not affected by the time dependence of the $Y_{i}^{\prime}$ 's and, thanks to the strict stationarity of $\left\{Y_{i}\right\}_{i \in \mathbb{N}}$, we have that $E[\bar{Y}]=\mu$ and $E\left[\left(\hat{\sigma}_{-}\right)^{2}\right]=\mu_{2}^{-}$. Concerning the variance of $\bar{Y}$, thanks to the strict stationarity of $\left\{Y_{i}\right\}_{i \in \mathbb{N}}$, we have that (see, e.g., Brockwell and Davis , 1991)

$$
\operatorname{Var}[\bar{Y}]=\frac{\sigma^{2}}{n}+\frac{2}{n} \sum_{i=1}^{n}\left(1-\frac{i}{n}\right) \sigma_{i}
$$


The variance of $\left(\hat{\sigma}_{-}\right)^{2}$ is given by:

$$
\begin{aligned}
\operatorname{Var}\left[\left(\hat{\sigma}_{-}\right)^{2}\right] & =\operatorname{Var}\left[\frac{1}{n} \sum_{i=1}^{n} Y_{i}^{2} \mathbb{I}_{0}\left(Y_{i}\right)\right] \\
& =\frac{1}{n^{2}} \operatorname{Var}\left[\sum_{i=1}^{n} Y_{i}^{2} \mathbb{I}_{0}\left(Y_{i}\right)\right] \\
& =\frac{1}{n} \operatorname{Var}\left[Y_{1}^{2} \mathbb{I}_{0}\left(Y_{1}\right)\right]+\frac{2}{n^{2}} \sum_{i=1}^{n} \sum_{j=i+1}^{n} \operatorname{Cov}\left[Y_{i}^{2} \mathbb{I}_{0}\left(Y_{i}\right), Y_{j}^{2} \mathbb{I}_{0}\left(Y_{j}\right)\right] \\
& =\frac{\mu_{4}^{-}-\left(\mu_{2}^{-}\right)^{2}}{n}+\frac{2}{n} \sum_{i=1}^{n}\left(1-\frac{i}{n}\right) \operatorname{Cov}\left[Y_{1}^{2} \mathbb{I}_{0}\left(Y_{1}\right), Y_{i+1}^{2} \mathbb{I}_{0}\left(Y_{i+1}\right)\right] \\
& =\frac{\mu_{4}^{-}-\left(\mu_{2}^{-}\right)^{2}}{n}+\frac{2}{n} \sum_{i=1}^{n}\left(1-\frac{i}{n}\right) \sigma_{2, i}^{-} .
\end{aligned}
$$

Now, we obtain the covariance between the two random variables involved in (5).

$$
\begin{aligned}
\operatorname{Cov}\left[\bar{Y} ;\left(\hat{\sigma}^{-}\right)^{2}\right] & =\frac{1}{n^{2}} \sum_{i=1}^{n} \sum_{j=1}^{n} \operatorname{Cov}\left(Y_{i} ; Y_{j}^{2} \mathbb{I}_{0}\left(Y_{j}\right)\right) \\
& =\frac{1}{n^{2}} \sum_{i=1}^{n} \operatorname{Cov}\left(Y_{i} ; Y_{i}^{2} \mathbb{I}_{0}\left(Y_{i}\right)\right)+\frac{1}{n^{2}} \sum_{i=1}^{n} \sum_{i \neq j=1}^{n} \operatorname{Cov}\left(Y_{i} ; Y_{j}^{2} \mathbb{I}_{0}\left(Y_{j}\right)\right) \\
& =\frac{\mu_{3}^{-}-\mu \mu_{2}^{-}}{n}+\frac{1}{n} \sum_{i=1}^{n-1}\left(1-\frac{i}{n}\right)\left(\sigma_{1,2, i}^{-}+\sigma_{2,1, i}^{-}\right)
\end{aligned}
$$

where

$$
\sigma_{1,2, i}^{-}=\operatorname{Cov}\left(Y_{1} ; Y_{i+1}^{2} \mathbb{I}_{0}\left(Y_{i+1}\right)\right)=E\left[Y_{1} ; Y_{i+1}^{2} \mathbb{I}_{0}\left(Y_{i+1}\right)\right]-\mu \mu_{2}^{-}
$$

and

$$
\sigma_{2,1, i}^{-}=\operatorname{Cov}\left(Y_{i+1} ; Y_{1}^{2} \mathbb{I}_{0}\left(Y_{1}\right)\right)=E\left[Y_{i+1} ; Y_{1}^{2} \mathbb{I}_{0}\left(Y_{1}\right)\right]-\mu \mu_{2}^{-} \quad:
$$

The asymptotic variance covariance covariance matrix of the vector (5) is then given by

$$
\tilde{\boldsymbol{\Sigma}}=\boldsymbol{\Sigma}+\lim _{n \rightarrow \infty}\left[\begin{array}{cc}
2 \sum_{i=1}^{n}\left(1-\frac{i}{n}\right) \sigma_{i} & +\sum_{i=1}^{n}\left(1-\frac{i}{n}\right)\left(\sigma_{1,2, i}^{-}+\sigma_{2,1, i}^{-}\right) \\
\sum_{i=1}^{n}\left(1-\frac{i}{n}\right)\left(\sigma_{1,2, i}^{-}+\sigma_{2,1, i}^{-}\right) & 2 \sum_{i=1}^{n}\left(1-\frac{i}{n}\right) \sigma_{2, i}^{-}
\end{array}\right] .
$$

Note that the matrix $\tilde{\boldsymbol{\Sigma}}$ coincides with the matrix $\boldsymbol{\Sigma}$ plus a term reflecting the time dependence among subsequent excess returns. Now, it is worthwhile to note that if $\left\{X_{t}\right\}_{t \in \mathbb{N}}$ is assumed stationary and $\alpha$-mixing of size $-\frac{2+\delta}{\delta}$, then Proposition 1 and Proposition 2 assure that:

$$
\begin{aligned}
& \sum_{\substack{i=1 \\
\infty}}^{\infty}\left|\sigma_{i}\right|<\infty \\
& \sum_{i=1}^{\infty}\left|\sigma_{2, i}^{-}\right|<\infty
\end{aligned}
$$


Moreover, the above expressions and the $\alpha$-mixing assumption assure also that:

$$
\sum_{i=1}^{\infty}\left|\sigma_{1,2, i}^{-}+\sigma_{2,1, i}^{-}\right|<\infty
$$

Now, if (12) and (13) hold, then

$$
\begin{aligned}
& \lim _{n \rightarrow \infty} \sum_{i=1}^{n}\left(1-\frac{i}{n}\right) \sigma_{i}=\sum_{i=1}^{\infty} \sigma_{i} \\
& \lim _{n \rightarrow \infty} \sum_{i=1}^{n}\left(1-\frac{i}{n}\right)\left(\sigma_{1,2, i}^{-}+\sigma_{2,1, i}^{-}\right)=\sum_{i=1}^{\infty}\left(\sigma_{1,2, i}^{-}+\sigma_{2,1, i}^{-}\right) \\
& \lim _{n \rightarrow \infty} \sum_{i=1}^{n}\left(1-\frac{i}{n}\right) \sigma_{2, i}^{-}=\sum_{i=1}^{\infty} \sigma_{2, i}^{-}
\end{aligned}
$$

and Corollary 1 can be specialized as follows.

Corollary 3 Let $\left\{Y_{t}\right\}_{t \in \mathbb{Z}}$ be stationary and $\alpha$-mixing of size $-\frac{2+\delta}{\delta}$ with $0<\delta<\infty$ and $E\left[\left|Y_{t}\right|^{4+\delta}\right]<\infty$. Then

$$
\sqrt{n}\left[\begin{array}{c}
\bar{Y}-\mu \\
\left(\hat{\sigma}^{-}\right)^{2}-\mu_{2}^{-}
\end{array}\right] \stackrel{d}{\longrightarrow} \mathbf{B N}(\mathbf{0} ; \tilde{\Sigma})
$$

where

$$
\tilde{\boldsymbol{\Sigma}}=\boldsymbol{\Sigma}+\left[\begin{array}{cc}
2 \sum_{i=1}^{\infty} \sigma_{i} & \sum_{i=1}^{\infty}\left(\sigma_{1,2, i}^{-}+\sigma_{2,1, i}^{-}\right) \\
\sum_{i=1}^{\infty}\left(\sigma_{1,2, i}^{-}+\sigma_{2,1, i}^{-}\right) & 2 \sum_{i=1}^{\infty} \sigma_{2, i}^{-}
\end{array}\right] \text {. }
$$

The following proposition follows again from an application of the delta method.

Proposition 3 Under the assumption of Corollary 3, it follows that

$$
\sqrt{n}(\widehat{\Upsilon}-v) \stackrel{d}{\longrightarrow} \mathcal{N}\left(0 ; \tilde{V}_{v}\right)
$$

where

$$
\tilde{V}_{v}=V_{v}+2 \sum_{i=1}^{\infty} \frac{\sigma_{i}}{\mu_{2}^{-}}-v \sum_{i=1}^{\infty} \frac{\left(\sigma_{1,2, i}^{-}+\sigma_{2,1, i}^{-}\right)}{\left(\mu_{2}^{-}\right)^{3 / 2}}+\frac{v^{2}}{2} \sum_{i=1}^{\infty} \frac{\sigma_{2, i}^{-}}{\left(\mu_{2}^{-}\right)^{2}}
$$

As suggested by Lo (2002) in the analysis of the Sharpe Ratio, under some additional regularity assumptions (see Newey and West , 1987), the variance $\tilde{V}_{v}$ can be consistently estimated starting from the Newey-West estimator $\widehat{\tilde{\Sigma}}_{N W}$ of $\tilde{\Sigma}$ as follows:

$$
\widehat{\tilde{V}}_{v}=\left[\begin{array}{c}
\frac{1}{\hat{\sigma}^{-}} \\
-\frac{\bar{Y}}{2\left(\hat{\sigma}^{-}\right)^{3 / 2}}
\end{array}\right]^{\prime} \widehat{\tilde{\Sigma}}_{N W}\left[\begin{array}{c}
\frac{1}{\hat{\sigma}^{-}} \\
-\frac{\bar{Y}}{2\left(\hat{\sigma}^{-}\right)^{3 / 2}}
\end{array}\right] .
$$


Finally, the following asymptotic $(1-\alpha)$-CI for $v$ can be introduced:

$$
\left(\widehat{\Upsilon}-z_{1-\frac{\alpha}{2}} \sqrt{\frac{\hat{\tilde{V}}_{v}}{n}} ; \widehat{\Upsilon}+z_{1-\frac{\alpha}{2}} \sqrt{\frac{\widehat{\tilde{V}}_{v}}{n}}\right) .
$$

\section{$5 \quad$ Asymptotic distribution of $\widehat{\Omega}$ and confidence interval for $\omega$}

\subsection{The case of i.i.d. returns}

As for the Sortino Ratio, we first assume that the sample excess returns $Y_{1}, Y_{2}, \ldots, Y_{n}$ are i.i.d. and we search for the large sample distribution of the random vector

$$
\left[\begin{array}{c}
\bar{Y} \\
\hat{\delta}^{-}
\end{array}\right]=\frac{1}{n} \sum_{i=1}^{n}\left[\begin{array}{c}
Y_{i} \\
-Y_{i} \mathbb{I}_{0}\left(Y_{i}\right)
\end{array}\right] .
$$

With this aim, we first obtain the expectation and the asymptotic variance-covariance matrix of (15). The algebraic passages followed in this analysis are very similar to those followed in the analysis of the Sortino Ratio. For this reason, in this section we provide only the main results.

The expectation and variance of $\bar{Y}$ are reported in (6). The expectation of $\hat{\delta}^{-}$is given by:

$$
E\left[\hat{\delta}^{-}\right]=-\int_{-\infty}^{0} y f_{Y}(y) d y=-\mu^{-}
$$

Thanks to the independence and the identical distribution of the $Y_{i}$ 's, we have that:

$$
\begin{gathered}
\operatorname{Var}\left[\hat{\delta}^{-}\right]=\frac{1}{n}\left[\int_{-\infty}^{0} y^{2} f_{Y}(y) d y-\left(\mu^{-}\right)^{2}\right]=\frac{\mu_{2}^{-}-\left(\mu^{-}\right)^{2}}{n} ; \\
\operatorname{Cov}\left(\bar{Y} ; \hat{\delta}^{-}\right)=\frac{\left(\mu \cdot \mu^{-}-\mu_{2}^{-}\right)}{n} .
\end{gathered}
$$

Thank to the multivariate central limit theorem, and to expression (6), (16), (17), and (18), we can state the following theorem.

Theorem 3 Let $Y$ a random variable with distribution function $F$ and assume that $E\left[Y^{j}\right] \leq$ $\infty$ for $j=1,2$. Let $Y_{1}, \ldots, Y_{n}$ ne an i.i.d. sample from $F$. It follows that

$$
\sqrt{n}\left[\begin{array}{c}
\bar{Y}-\mu \\
\hat{\delta}^{-}+\mu^{-}
\end{array}\right] \stackrel{d}{\longrightarrow} \mathcal{N}(\mathbf{0}, \boldsymbol{\Xi})
$$

where BN means "bivariate normal",

$$
\mathbf{0}=\left[\begin{array}{l}
0 \\
0
\end{array}\right], \quad \text { and } \quad \boldsymbol{\Xi}=\left[\begin{array}{cc}
\sigma^{2} & \mu \cdot \mu^{-}-\mu_{2}^{-} \\
\mu \cdot \mu^{-}-\mu_{2}^{-} & \mu_{2}^{-}-\left(\mu^{-}\right)^{2}
\end{array}\right] \text {. }
$$


By applying the delta method, it possible to prove the following corollary.

Corollary 4 If the assumptions of Theorem 2 are satisfied, it results that

$$
\sqrt{n}(\widehat{\Omega}-\omega) \stackrel{d}{\longrightarrow} \mathcal{N}\left(0 ; V_{\omega}\right)
$$

where

$$
V_{\omega}=\frac{\sigma^{2}}{\left(\mu^{-}\right)^{2}}-2\left(\frac{\mu \cdot \mu^{-}-\mu_{2}^{-}}{\left(\mu^{-}\right)^{2}}\right) \omega+\left(\frac{\mu_{2}^{-}-\left(\mu^{-}\right)^{2}}{\left(\mu^{-}\right)^{2}}\right) \omega^{2}
$$

A consistent estimator for the variance $V_{\omega}$ is

$$
\widehat{V}_{\omega}=\frac{S^{2}}{\left(\hat{\mu}^{-}\right)^{2}}-2\left(\frac{\bar{Y} \hat{\mu}^{-}-\hat{\mu}_{2}^{-}}{\left(\hat{\mu}^{-}\right)^{2}}\right) \widehat{\Omega}+\left(\frac{\hat{\mu}_{2}^{-}-\left(\hat{\mu}^{-}\right)^{2}}{\left(\hat{\mu}^{-}\right)^{2}}\right) \widehat{\Omega}^{2}
$$

and the following asymptotic $(1-\alpha)$-Confidence Interval (CI) for $\omega$ can be obtained by applying the standard analytical inversion method:

$$
\left(\widehat{\Omega}-z_{1-\frac{\alpha}{2}} \sqrt{\frac{\widehat{V}_{\omega}}{n}} ; \widehat{\Omega}+z_{1-\frac{\alpha}{2}} \sqrt{\frac{\widehat{V}_{\omega}}{n}}\right) .
$$

\subsection{The general case of not-i.i.d. returns}

As done for the Sortino Ratio, we first derive the variance-covariance matrix of the random vector (15). As in the previous subsection, here we report only the main results, avoiding several algebraic passages.

The variance of $\bar{Y}$ is given by (11). The variance of $\hat{\delta}^{-}$is:

$$
\begin{aligned}
\operatorname{Var}\left[\hat{\delta}^{-}\right] & =\frac{\mu_{2}^{-}-\left(\mu^{-}\right)^{2}}{n}+\frac{2}{n} \sum_{i=1}^{n}\left(1-\frac{i}{n}\right) \operatorname{Cov}\left[Y_{1} \mathbb{I}_{0}\left(Y_{1}\right), Y_{i+1} \mathbb{I}_{0}\left(Y_{i+1}\right)\right] \\
& =\frac{\mu_{2}^{-}-\left(\mu^{-}\right)^{2}}{n}+\frac{2}{n} \sum_{i=1}^{n}\left(1-\frac{i}{n}\right) \sigma_{i}^{-}
\end{aligned}
$$

The covariance between $\bar{Y}$ and $\hat{\delta}^{-}$results:

$$
\operatorname{Cov}\left[\bar{Y} ; \hat{\delta}^{-}\right]=\frac{\mu \mu^{-}-\mu_{2}^{-}}{n}-\frac{1}{n} \sum_{i=1}^{n-1}\left(1-\frac{i}{n}\right)\left(\sigma_{1, i}^{-}+\sigma_{i, 1}^{-}\right)
$$

where

$$
\sigma_{1, i}^{-}=\operatorname{Cov}\left(Y_{1} ; Y_{i+1} \mathbb{I}_{0}\left(Y_{i+1}\right)\right)=E\left[Y_{1} ; Y_{i+1} \mathbb{I}_{0}\left(Y_{i+1}\right)\right]-\mu \mu^{-}
$$

and

$$
\sigma_{i, 1}^{-}=\operatorname{Cov}\left(Y_{i+1} ; Y_{1} \mathbb{I}_{0}\left(Y_{1}\right)\right)=E\left[Y_{i+1} ; Y_{1} \mathbb{I}_{0}\left(Y_{1}\right)\right]-\mu \mu^{-}
$$


The asymptotic variance-covariance matrix of the random vector (15) is then given by:

$$
\tilde{\boldsymbol{\Xi}}=\boldsymbol{\Xi}+\lim _{n \rightarrow \infty}\left[\begin{array}{cc}
2 \sum_{i=1}^{n}\left(1-\frac{i}{n}\right) \sigma_{i} & -\sum_{i=1}^{n}\left(1-\frac{i}{n}\right)\left(\sigma_{1, i}^{-}+\sigma_{i, 1}^{-}\right) \\
-\sum_{i=1}^{n}\left(1-\frac{i}{n}\right)\left(\sigma_{1, i}^{-}+\sigma_{i, 1}^{-}\right) & 2 \sum_{i=1}^{n}\left(1-\frac{i}{n}\right) \sigma_{i}^{-}
\end{array}\right]
$$

As explained in detail in Section..., if $\left\{Y_{t}\right\}_{t \in \mathbb{N}}$ is assumed stationary and $\alpha$-mixing of size $-\frac{2+\delta}{\delta}$ with $\delta>0$ and $E\left[\left|Y_{t}\right|^{2+\delta}\right]<\infty$, then

$$
\begin{aligned}
& \sum_{i=1}^{\infty}\left|\sigma_{i}\right|<\infty, \\
& \sum_{\substack{i=1 \\
\infty}}^{\infty}\left|\sigma_{1, i}^{-}+\sigma_{i, 1}^{-}\right|<\infty, \\
& \sum_{i=1}^{\infty}\left|\sigma_{i}^{-}\right|<\infty,
\end{aligned}
$$

and, consequently

$$
\begin{aligned}
& \lim _{n \rightarrow \infty} \sum_{i=1}^{n}\left(1-\frac{i}{n}\right) \sigma_{i}=\sum_{i=1}^{\infty} \sigma_{i} \\
& \lim _{n \rightarrow \infty} \sum_{\substack{n=1 \\
n}}^{n}\left(1-\frac{i}{n}\right)\left(\sigma_{1, i}^{-}+\sigma_{i, 1}^{-}\right)=\sum_{i=1}^{\infty}\left(\sigma_{1, i}^{-}+\sigma_{i, 1}^{-}\right), \\
& \lim _{n \rightarrow \infty} \sum_{i=1}^{n}\left(1-\frac{i}{n}\right) \sigma_{i}^{-}=\sum_{i=1}^{\infty} \sigma_{i}^{-} .
\end{aligned}
$$

Then, Corollary 1 can be specialized as follows.

Corollary 5 Let $\left\{Y_{t}\right\}_{t \in \mathbb{Z}}$ be stationary and $\alpha$-mixing of size $-\frac{2+\delta}{\delta}$ with $0<\delta<\infty$ and $E\left[\left|Y_{t}\right|^{2+\delta}\right]<\infty$. Then

$$
\sqrt{n}\left[\begin{array}{c}
\bar{Y}-\mu \\
\hat{\delta}^{-}+\mu^{-}
\end{array}\right] \stackrel{d}{\longrightarrow} \mathbf{B N}(\mathbf{0} ; \tilde{\Xi})
$$

where

$$
\tilde{\boldsymbol{\Xi}}=\boldsymbol{\Xi}+\left[\begin{array}{cc}
2 \sum_{i=1}^{\infty} \sigma_{i} & -\sum_{i=1}^{\infty}\left(\sigma_{1, i}^{-}+\sigma_{i, 1}^{-}\right) \\
-\sum_{i=1}^{\infty}\left(\sigma_{1, i}^{-}+\sigma_{i, 1}^{-}\right) & 2 \sum_{i=1}^{\infty} \sigma_{i}^{-}
\end{array}\right]
$$

From Corollary 5 the following proposition follows.

Proposition 4 Under the assumption of Corollary 5, it follows that

$$
\sqrt{n}(\widehat{\Omega}-\omega) \stackrel{d}{\longrightarrow} \mathcal{N}\left(0, \breve{V}_{\omega}\right)
$$

where

$$
\tilde{V}_{\omega}=V_{\omega}+2 \sum_{i=1}^{\infty} \frac{\sigma_{i}}{\left(\mu^{-}\right)^{2}}+2 \omega \sum_{i=1}^{\infty} \frac{\left(\sigma_{1, i}^{-}+\sigma_{i, 1}^{-}\right)}{\left(\mu^{-}\right)^{2}}+2 \omega^{2} \sum_{i=1}^{\infty} \frac{\sigma_{i}^{-}}{\left(\mu^{-}\right)^{2}}
$$


Under some additional regularity assumptions (see Newey and West, 1987), the variance $\tilde{V}_{\omega}$ can be consistently estimated starting from the Newey-West estimator $\widehat{\widetilde{\Xi}}_{N W}$ of $\tilde{\boldsymbol{\Xi}}$ as follows:

$$
\widehat{\tilde{V}}_{\omega}=\left[\begin{array}{c}
\frac{1}{\hat{\delta}^{-}} \\
-\frac{\bar{Y}}{\left(\hat{\delta}^{-}\right)^{2}}
\end{array}\right]^{\prime} \widehat{\widetilde{\Xi}}_{N W}\left[\begin{array}{c}
\frac{1}{\hat{\delta}^{-}} \\
-\frac{\bar{Y}}{\left(\hat{\delta}^{-}\right)^{2}}
\end{array}\right] .
$$

Then the following asymptotic $(1-\alpha)$-CI for $\omega$ can be introduced:

$$
\left(\widehat{\Omega}-z_{1-\frac{\alpha}{2}} \sqrt{\frac{\widehat{\tilde{V}}_{\omega}}{n}} ; \widehat{\Omega}+z_{1-\frac{\alpha}{2}} \sqrt{\frac{\widehat{\tilde{V}}_{\omega}}{n}}\right) .
$$

\section{Design of the simulation study}

In the present section it is described the simulation study we perform to evaluate the properties of the large sample confidence intervals (10), (14), (19) and (20). Under the assumption of i.i.d. returns we replicate the simulation study performed in De Capitani and Zenga (2011). In the general case of not-i.i.d. returns the design of simulations is taken from De Capitani (2010). The confidence interval for the Sharpe Ratio $\psi$ is also computed in order to provide a useful cornerstone for the comment of the results. The aforementioned confidence interval for $\psi$ can be obtained from the results in Lo (2002) both under the i.i.d. assumption and under the not-i.i.d assumption (for a re-examination of their derivation and a study of their properties we refer the interested reader to De Capitani and Zenga , 2011; De Capitani , 2010).

\section{Design of the simulation study under the i.i.d. assumption}

- sample sizes: 50, 100, 200, 400, 800;

- return distributions: Normal; Laplace; Student's $t$ with 3 and 5 degrees of freedom; Skew Normal with low/high, positive/negative asymmetry; Skew $t$ with 5 degrees of freedom and low/high, positive/negative asymmetry. A brief summary of the main features of the considered distribution is given in Table 1 where the values of the asymmetry indicator $\gamma_{1}=E\left[(X-\mu)^{3} / \sigma^{3}\right]$ and Pearson's kurtosis $\gamma_{2}=E\left[(X-\mu)^{4} / \sigma^{4}\right]-$ 3 are given.

- nominal coverages of the large sample CIs: 0.9, 0.95, 0.975, 0.99;

- value of the (daily) target return $k$ : 0.000068 (which correspond to a annual rate of return of about $2.5 \%$ )

- value of the standard deviation $\sigma$ of the excess return $Y: \sigma=0.01, \sigma=0.05, \sigma=0.1$. The different values of $\sigma$ are chosen coherently with the values of the standard deviation of the daily returns of the equities in the S\&P 100 along the period 2005-2007.

- true values of $\psi, v$ and $\omega$ : for each value of $\sigma$ we consider 3 different values of $\mu_{Y}=$ $E[Y]=E[X]-k$. The lowest value gives rise to a Sharpe Ratio of 0.05; the highest 


\begin{tabular}{cccccc}
\hline Distribution & $\gamma_{1}$ & $\gamma_{2}$ & Distribution & $\gamma_{1}$ & $\gamma_{2}$ \\
\hline Normal & 0 & 0 & Laplace & 0 & 3 \\
$t$ with 3 df & 0 & $\infty$ & $t$ with 5 df & 0 & 6 \\
Skew N. (High as.) & \pm 0.6670 & 0.5098 & Skew N. (Low as.) & \pm 0.4538 & 0.3051 \\
Skew $t$ (High as.) & \pm 1.0758 & 8.9208 & Skew $t$ (Low as.) & \pm 0.5527 & 6.8020 \\
\hline
\end{tabular}

Table 1: Values of $\gamma_{1}$ and $\gamma_{2}$ for the 12 distributions considered.

value gives rise to a Sharpe Ratio of 0.5 ; the remaining value corresponds to a Sharpe Ratio of 0.25 . As for the values of $\sigma$ the three different values of $\psi$ are chosen on the basis of the daily Sharpe Ratios of the equities in the S\&P 100 along the period 2005-2007. The three different values of $v$ and $\omega$ are determined by the different combinations of $\mu$ and $\sigma$ according to the particular distribution under investigation.

- number of replications: $5 \times 10^{4}$

\section{Design of the simulation study in the not-i.i.d. case}

- data generating process: we consider a $\operatorname{GARCH}(1,1)$ process with symmetric innovations and finite fourth moment. In more detail:

$$
X_{t}-\mu_{X}=\sigma_{t} \epsilon_{t} \quad \text { where } \quad \sigma_{t}^{2}=0.001+0.1\left(X_{t-1}-\mu_{X}\right)^{2}+0.8 \sigma_{t-1}^{2},
$$

and $\epsilon_{t}(t=1,2, \ldots)$ i.i.d., symmetrically distributed and with $E\left[\epsilon_{t}\right]=0, E\left[\epsilon_{t}^{2}\right]=1$ and $E\left[\epsilon_{t}^{4}\right]<\infty$. Note that this process satisfies the regularity conditions necessary to obtain the confidence intervals (14) and (20) since, as proved in Carrasco and Chen (2002), it is $\beta$-mixing with exponential decay and, consequently it is also $\alpha$-mixing of size $-\frac{2+\delta}{\delta}$ for some $\delta>0$. According to the simulations design under the i.i.d. assumption, we consider three different values of $\mu_{X}$ : 0.005068, 0.025068, 0.050068 . The values of the Sharpe Ratio associated to the three values of $\mu_{X}$ are, respectively, $0.05,0.025$ and 0.5 .

- Distribution of $\epsilon_{t}$ : Standard normal distribution, Laplace distribution with unit variance and zero mean, Student's $t$ distribution with 5 degrees of freedom rescaled by the factor $(3 / 5)^{0.5}$ (in this way, the resulting distribution has zero mean and unit variance).

- sample sizes: 50, 100, 200, 400, 800, 1600, 3200;

- nominal coverages of the large sample CIs: 0.9, 0.95, 0.975, 0.99;

- number of replications: $5 \times 10^{4}$.

Concluding, we highlight that the Newey-West estimator $\widehat{\tilde{\Sigma}}_{N W}$ and $\widehat{\tilde{\Xi}}_{N W}$, necessary to define the confidence intervals (14) and (20), are obtained determining the value of the bandwidth $m$ in three different way:

- naive method: deterministic bandwidth given by $m=5 \times n^{1 / 4}$; 
- Newey and West's procedure (NW-bw): automatic and "data dependent" selection procedure proposed in Newey and West (1994) and clearly outlined in Hall (2005), pp. 82-83.

- Andrews and Monahan's procedure (PR-bw): automatic and "data dependent" selection procedure proposed in Andrews and Monahan (1992) and based on the so-called "pre-whitening and recoloring" technique (see, again, the clear presentation in Hall , 2005, pp. 84-85).

\section{$7 \quad$ Results}

\subsection{Independent and identically distributed returns}

Here, we do not give all the detailed results of the simulation study. Only a brief summary is given in order to emphasize the main aspects: the impact of asymmetry and kurtosis of the parent distribution on the coverage accuracy of the large sample confidence intervals. The minimum sample sizes assuring a sufficient adherence of the actual coverage to the nominal one is determined by the criterion described below. It is well known that the $t$ distribution approaches the Normal distribution when the df increase. Further, it is common to retain that the $t$ distribution with $30 \mathrm{df}$ is approximated by the Normal distribution very well. As a consequence, when sampling from the Normal distribution, the asymptotic CI

$$
\left(\bar{X}-z_{1-\alpha / 2} \sqrt{S^{2} / n} ; \bar{X}+z_{1-\alpha / 2} \sqrt{S^{2} / n}\right)
$$

is considered accurate if $n \geq 30$. The actual coverages of the above CI when $n=30$ (reported in Table 2) can, then, be adopted as a cornerstone to determine the aforementioned minimum sample sizes. In more detail, we will consider the simulated coverages sufficiently close to their nominal value $(1-\alpha)$ if they belong to the interval $\left(1-\alpha-\epsilon_{\alpha} ; 1-\alpha+\epsilon_{\alpha}\right)$, where $\epsilon_{\alpha}$ is defined in Table 2 . In Table 3 the minimum sample sizes obtained applying this criterion are given. To give an example, the sample size 50 corresponding to "performance ratio = Sharpe Ratio", "parent distribution $=\mathbf{N}$ ", "Value of $\psi=$ small", " $100(1-\alpha) \%=90 \% "$ means that, when samplig from the Normal distribution, a sample size of 50 can be considered sufficient for the asymptotic confidence interval at nominal confidence level $90 \%$ whether is the value of $\sigma$ among $(0.01,0.05,0.1)$. In general, the simulations highlight that:

- the fatter the tails of the parent distribution, the worst the coverage accuracy of the confidence interval for all the three performance measures. In more detial, the CIs for $\psi$ and $v$ are very sensible to the increase in the fatness of the tails while the confidence interval for $\omega$ shows a substantial robustness with respect to this feature of the parent distribution. It is worthwhile to note that if the tails of the parent distribution are so heavy that the existence of fourth moment is not assured (Student's $t$ with $3 \mathrm{df}$ ), the CIs for the Sharpe and Omega Ratio are not defined since the asymptotic theory on which they are based requires the existence of the moments up to the fourth order. Nevertheless they can be calculated even when sampling from the Student's $t$ with 3 df since the fourth sample moment is always finite. However, these CIs are incorrect and, ideed, the simulations show that their coverage accuracy is bad for all the sample 


\begin{tabular}{lcccc}
\hline Nominal Coverage: $(1-\alpha)$ & 0.9 & 0.95 & 0.975 & 0.99 \\
\hline Actual Coverage: $a_{\alpha}$ & 0.8896 & 0.9407 & 0.9674 & 0.9848 \\
$\epsilon_{\alpha}=\left|(1-\alpha)-a_{\alpha}\right|$ & 0.0104 & 0.0093 & 0.0076 & 0.0052 \\
$1-\alpha+\epsilon_{\alpha}$ & 0.9104 & 0.9593 & 0.9826 & 0.9952 \\
$1-\alpha-\epsilon_{\alpha}$ & 0.8896 & 0.9407 & 0.9674 & 0.9848 \\
\hline
\end{tabular}

Table 2: Comparison between the nominal and actual coverage probabilities of the asymptotic CI (21)

sizes. On the contrary, the CI for $\omega$ is well defined for all the parent distributions with second moment and the simulations show that, when sampling from $T_{3}$ a sample size of 400 is sufficient. This result is coherent with those provided in De Capitani and Zenga (2011) for the MAD and MD ratios.

- the coverage accuracy of the CI for $\psi$ is better when the parent distribution is positively skewed. On the contrary the coverage accuracy of the CIs for $v$ and $\omega$ slightly improves for negatively skewed distributions. Also in this case the confidence interval for $\omega$ is the most robust whit respect to the changes in the asymmetry of the parent distribution.

- the higher is the true value of the ratio, the worst is the coverage accuracy. Again, this effect is more evident for the CIs for $\psi$ and $v$ and sligtly visible concerning the CI for $\omega$.

- Globally, a sample size of 800 is sufficient for the CIs for $\psi$ and $v$ in all the cases investigated with the relevant exception of the Student's $t$ with 3 df. On the contrary a sample size of 400 is always sufficient for the CI for $\omega$ even if the convergence of the actual coverage to the nominal one is, for this CI, slower in the more "regular" simulation settings (such as those concerning the Normal, Laplace, Student's $t$ with 5 df or the Skew Normal).

\subsection{Dependent identically distributed returns}

In tables 4, 5 and 6 we give the simulated coverages of the large sample CIs for the Sharpe Ratio (see De Capitani , 2010), Sortino Ratio (see expression 14) and Omega Ratio (see expression 20), respectively. In these tables the simulated coverages sufficiently adherent to the nominal coverages (in accordance to the criterion described in the previous section) are highlighted coloring the background of the corresponding cells in gray. As it is evident from the tables, the presence of the time dependence has a great impact on the coverage accuracy of the asymptotic CIs. Some of the features of the CIs derived under the i.i.d. assumption can be observed also in this this context with a greater evidence. In detail:

- The dependence of the coverage accuracy on the true value of the ratio is, in this context, very strong. Also the CI for $\omega$ is now affected by this effect even if, under the i.i.d. assumption only a slight impact was observed. 


\begin{tabular}{|c|c|c|c|c|c|c|c|c|c|c|c|c|}
\hline \multicolumn{13}{|c|}{ SHARPE RATIO } \\
\hline \multirow{2}{*}{$\begin{array}{c}\text { Value of } \psi \\
100(1-\alpha) \%\end{array}$} & \multicolumn{4}{|c|}{ small } & \multicolumn{4}{|c|}{ medium } & \multicolumn{4}{|c|}{ high } \\
\hline & $90 \%$ & $95 \%$ & $97.5 \%$ & $99 \%$ & $90 \%$ & $95 \%$ & $97.5 \%$ & $99 \%$ & $\overline{90 \%}$ & $95 \%$ & $97.5 \%$ & $99 \%$ \\
\hline $\mathbf{N}$ & 50 & 50 & 50 & 50 & 50 & 50 & 50 & 50 & 100 & 50 & 50 & 50 \\
\hline $\mathbf{L}$ & 200 & 100 & 100 & 100 & 200 & 200 & 200 & 200 & 200 & 200 & 200 & 200 \\
\hline$T_{5}$ & 100 & 100 & 100 & 100 & 200 & 200 & 200 & 100 & 400 & 400 & 400 & 200 \\
\hline$T_{3}$ & - & - & - & - & - & - & - & - & - & - & - & - \\
\hline $\mathrm{SNL}+$ & 50 & 50 & 50 & 50 & 50 & 50 & 50 & 50 & 50 & 50 & 50 & 50 \\
\hline $\mathrm{SNH}+$ & 50 & 50 & 50 & 50 & 50 & 50 & 50 & 50 & 50 & 50 & 50 & 50 \\
\hline STL+ & 100 & 100 & 100 & 100 & 200 & 200 & 100 & 100 & 400 & 200 & 200 & 200 \\
\hline STH+ & 50 & 100 & 100 & 100 & 50 & 100 & 50 & 50 & 200 & 200 & 100 & 200 \\
\hline SNL- & 50 & 50 & 50 & 50 & 50 & 50 & 50 & 50 & 100 & 100 & 50 & 100 \\
\hline SNH- & 50 & 50 & 50 & 50 & 50 & 50 & 50 & 50 & 100 & 100 & 100 & 50 \\
\hline STL- & 100 & 100 & 100 & 200 & 400 & 200 & 200 & 200 & 800 & 400 & 400 & 200 \\
\hline STH- & 200 & 200 & 200 & 200 & 400 & 400 & 400 & 200 & 800 & 800 & 800 & 800 \\
\hline \multicolumn{13}{|c|}{ SORTINO RATIO } \\
\hline Value of $v$ & \multicolumn{4}{|c|}{ small } & \multicolumn{4}{|c|}{ medium } & \multicolumn{4}{|c|}{ high } \\
\hline $100(1-\alpha) \%$ & $90 \%$ & $95 \%$ & $97.5 \%$ & $99 \%$ & $90 \%$ & $95 \%$ & $97.5 \%$ & $99 \%$ & $90 \%$ & $95 \%$ & $97.5 \%$ & $99 \%$ \\
\hline $\mathbf{N}$ & 50 & 50 & 100 & 200 & 50 & 50 & 100 & 200 & 100 & 50 & 100 & 200 \\
\hline $\mathbf{L}$ & 50 & 100 & 200 & 400 & 100 & 200 & 200 & 400 & 400 & 400 & 400 & 400 \\
\hline$T_{5}$ & 50 & 100 & 200 & 200 & 400 & 400 & 400 & 400 & 800 & 800 & 800 & 800 \\
\hline$T_{3}$ & - & - & - & - & - & - & - & - & - & - & - & - \\
\hline SNL+ & 50 & 50 & 100 & 400 & 50 & 50 & 100 & 200 & 50 & 100 & 100 & 200 \\
\hline $\mathrm{SNH}+$ & 50 & 100 & 200 & 200 & 50 & 100 & 100 & 200 & 50 & 100 & 100 & 200 \\
\hline STL+ & 50 & 100 & 200 & 200 & 400 & 200 & 200 & 400 & 800 & 800 & 800 & 400 \\
\hline STH+ & 100 & 200 & 400 & 400 & 200 & 200 & 400 & 400 & 800 & 800 & 800 & 400 \\
\hline SNL- & 50 & 50 & 50 & 100 & 50 & 50 & 50 & 200 & 50 & 50 & 100 & 200 \\
\hline SNH- & 50 & 50 & 50 & 100 & 50 & 50 & 100 & 100 & 100 & 50 & 100 & 200 \\
\hline & 50 & 100 & 100 & 200 & 400 & 400 & 200 & 400 & 800 & 800 & 800 & 800 \\
\hline STH- & 50 & 100 & 100 & 200 & 800 & 800 & 400 & 400 & 800 & 800 & 800 & 800 \\
\hline
\end{tabular}

\begin{tabular}{|c|c|c|c|c|c|c|c|c|c|c|c|c|}
\hline \multirow{3}{*}{$\begin{array}{c}\text { Value of } \omega \\
100(1-\alpha) \%\end{array}$} & \multicolumn{8}{|c|}{ OMEGA RATIO } & \multirow{2}{*}{\multicolumn{4}{|c|}{ high }} \\
\hline & \multicolumn{4}{|c|}{ small } & \multicolumn{4}{|c|}{ medium } & & & & \\
\hline & $\overline{90 \%}$ & $95 \%$ & $97.5 \%$ & $99 \%$ & $\overline{90 \%}$ & $95 \%$ & $97.5 \%$ & $99 \%$ & $\overline{90 \%}$ & $95 \%$ & $97.5 \%$ & $99 \%$ \\
\hline $\mathbf{N}$ & 50 & 200 & 200 & 400 & 50 & 200 & 200 & 400 & 50 & 100 & 200 & 400 \\
\hline L & 100 & 200 & 400 & 400 & 50 & 200 & 400 & 400 & 50 & 200 & 400 & 400 \\
\hline$T_{5}$ & 50 & 200 & 400 & 400 & 50 & 200 & 400 & 400 & 50 & 200 & 400 & 400 \\
\hline$T_{3}$ & 200 & 400 & 400 & 400 & 50 & 400 & 400 & 400 & 50 & 400 & 400 & 400 \\
\hline SNL+ & 50 & 200 & 400 & 400 & 50 & 200 & 400 & 400 & 50 & 200 & 400 & 400 \\
\hline $\mathrm{SNH}+$ & 50 & 200 & 200 & 400 & 50 & 100 & 200 & 400 & 50 & 100 & 200 & 400 \\
\hline STL+ & 100 & 200 & 400 & 400 & 50 & 200 & 400 & 400 & 50 & 200 & 400 & 400 \\
\hline $\mathrm{STH}+$ & 100 & 400 & 400 & 400 & 50 & 200 & 400 & 400 & 50 & 200 & 400 & 400 \\
\hline SNL- & 50 & 100 & 400 & 400 & 50 & 100 & 200 & 400 & 50 & 100 & 200 & 400 \\
\hline SNH- & 50 & 100 & 200 & 400 & 50 & 100 & 200 & 400 & 50 & 100 & 400 & 400 \\
\hline STL- & 50 & 100 & 200 & 400 & 50 & 100 & 200 & 400 & 50 & 100 & 400 & 400 \\
\hline STH- & 50 & 100 & 200 & 400 & 50 & 100 & 200 & 400 & 50 & 100 & 400 & 400 \\
\hline
\end{tabular}

Table 3: Minimum sample sizes necessary to reach a good coverage accuracy for the CIs for Sharpe, Sortino and Omega Ratio under i.i.d. assumption.

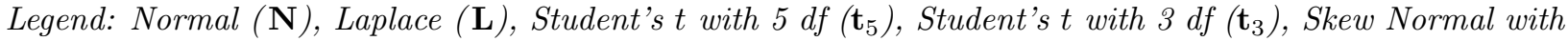
low positive skewness (SNL+), Skew Normal with high positive skewness (SNH+), Skew $t$ with low positive skewness ( $\mathbf{S t L}+$ ), Skew $t$ with high positive skewness ( $\mathbf{S t H}+$ ), Skew Normal with low negative skewness (SNL-), Skew Normal with high negative skewness (SNH-), Skew $t$ with low negative skewness 
- The simulation results highlight that the CIs obtained with the PR-bw and NW-bw methods for the determination of the bandwidth of the Newey-West estimator have a better coverage accuracy with respect to that obtained with the "naive" method. This result is rather expected and agrees with the evidence in Newey and West (1994). Moreover, we observe a substantial equivalence between PR-bw and NW-bw methods. This is (almost surely) due to the fact that the GARCH process has uncorrelated (but dependent) components and, as explained in Andrews and Monahan (1992) and recalled in Hall (2005) (see on page 83), the PR-bw should perform better than NW-bw when the data generating process exhibits a autoregressive behavior.

- In general, if the true value of the ratios is small and using the PR-bw method, a sample size between 400 and 800 can be considered sufficient for the CIs for the three ratio. On the contrary, when the true value of the ratios is medium, 800 is sufficient only in the case of Gaussian innovations. In the Laplace and Student's $t$ innovations cases, 3200 seems to be almost adequate only for the CI for $\omega$. Finally, if the true value of the ratios is high, a sample size of 3200 is not sufficient in any scenarios.

\section{Conclusions}

In this paper we derive a large sample confidence interval for the financial performance measures known as Sortino Ratio and Omega Ratio. These confidence intervals are obtained first under the strong assumption of independence and identical distribution of the returns and, later, generalized to the more general and realistic case in which returns follows a stationary and $\alpha$-mixing process. In order to evaluate the properties of the aforementioned CIs, a wide simulation study is performed both under the i.i.d. assumption and in the noti.i.d case. In the simulation, also the CIs for the Sharpe Ratio (obtained form the results in Lo , 2002, and De Capitani , 2010) is considered for a comparison. Evidence from the simulations suggests that the asymptotic confidence interval for the Omega Ratio seems to have the best features. It is worthwhile to note that the last is the only confidence interval that can be derived assuming the existence of "only" the second moment while the confidence intervals for the Sortino and Sharpe ratios require the existence of at least the fourth moment. This fact agrees with the results in De Capitani and Zenga (2011) where it is shown that the confidence intervals for the MAD an MD ratios, that are based on the existence of the second moment, are more accurate than the CI for the Sharpe Ratio. Simulation results emphasize that all CIs are very sensitive to the true value of the ratio: the grater is the value of the ratios, the worst is the coverage accuracy of the confidence intervals. Moreover, as expected, the fatter the tails of the returns distribution, the worst the coverage accuracy. However, the most meaningful result we obtain is that, under the more general assumption of not-i.i.d. returns, a sample size of 3200 is far to be generally sufficient for a good coverage accuracy of all the considered CIs. This is due mainly to the impact of the true value of the ratio on the convergence of the actual coverage to the nominal one and seriously affect the real applicability of the proposed confidence intervals. In fact, in the simulations we choose the true values of the ratios, the values of the standard deviation of the returns distribution and the parameters of the GARCH model coherently with the empirical evidence on daily 


\begin{tabular}{|c|c|c|c|c|c|c|c|c|c|c|c|c|c|}
\hline \multirow{2}{*}{\multicolumn{2}{|c|}{$\begin{array}{c}\text { Value of } \psi \\
100(1-\alpha) \%\end{array}$}} & \multicolumn{4}{|c|}{ small } & \multicolumn{4}{|c|}{ medium } & \multicolumn{4}{|c|}{ high } \\
\hline & & $\overline{90 \%}$ & $95 \%$ & $97.5 \%$ & $99 \%$ & & $95 \%$ & $97.5 \%$ & $99 \%$ & $\overline{90 \%}$ & $95 \%$ & $97.5 \%$ & $99 \%$ \\
\hline bw & n & \multicolumn{12}{|c|}{ ions } \\
\hline \multirow[t]{7}{*}{ PR-bw } & 50 & 84.39 & 90.69 & 93.87 & 96.41 & 83.20 & 89.51 & 93.22 & 96.10 & 79.92 & 86.42 & 91.03 & 94.43 \\
\hline & 100 & 87.46 & 93.11 & 96.27 & 98.19 & 86.31 & 92.09 & 95.44 & 97.63 & 83.50 & 89.71 & 93.55 & 96.35 \\
\hline & 200 & 88.48 & 93.87 & 96.52 & 98.17 & 87.33 & 92.78 & 95.78 & 98.06 & 83.71 & 90.32 & 94.30 & 97.10 \\
\hline & 400 & 89.47 & 94.37 & 97.01 & 98.69 & 88.31 & 93.65 & 96.50 & 98.30 & 85.67 & 91.92 & 95.19 & 97.41 \\
\hline & 800 & 90.23 & 95.43 & 97.58 & 99.01 & 89.45 & 94.35 & 96.95 & 98.68 & 86.04 & 92.34 & 95.64 & 97.81 \\
\hline & 1600 & 89.54 & 94.65 & 97.29 & 98.84 & 88.47 & 93.87 & 96.74 & 98.59 & 86.60 & 92.01 & 95.61 & 98.01 \\
\hline & 3200 & 89.67 & 94.64 & 97.24 & 98.77 & 88.99 & 94.09 & 97.02 & 98.63 & 86.97 & 92.98 & 96.11 & 98.08 \\
\hline \multirow[t]{7}{*}{ NW-bw } & 50 & 83.94 & 89.98 & 93.40 & 96.05 & 82.60 & 89.05 & 92.74 & 95.50 & 79.76 & 86.34 & 90.76 & 94.13 \\
\hline & 100 & 87.36 & 92.84 & 96.12 & 98.07 & 86.18 & 91.97 & 95.20 & 97.54 & 83.70 & 89.72 & 93.32 & 96.38 \\
\hline & 200 & 88.51 & 93.69 & 96.47 & 98.19 & 87.30 & 92.74 & 95.80 & 98.05 & 83.93 & 90.35 & 94.44 & 97.16 \\
\hline & 400 & 89.48 & 94.51 & 97.11 & 98.75 & 88.33 & 93.72 & 96.59 & 98.35 & 85.81 & 91.98 & 95.27 & 97.49 \\
\hline & 800 & 90.18 & 95.45 & 97.56 & 99.03 & 89.39 & 94.35 & 96.98 & 98.73 & 85.93 & 92.31 & 95.65 & 97.81 \\
\hline & 1600 & 89.57 & 94.70 & 97.30 & 98.87 & 88.48 & 93.85 & 96.77 & 98.62 & 86.55 & 92.05 & 95.54 & 97.99 \\
\hline & 3200 & 89.67 & 94.67 & 97.22 & 98.78 & 88.98 & 94.11 & 97.04 & 98.62 & 86.90 & 92.90 & 96.08 & 98.06 \\
\hline \multirow[t]{8}{*}{ naive } & 50 & 79.18 & 85.89 & 90.29 & 93.64 & 77.97 & 84.84 & 89.51 & 93.27 & 75.09 & 82.19 & 87.56 & 91.53 \\
\hline & 100 & 83.68 & 89.56 & 93.45 & 96.24 & 82.44 & 88.91 & 92.84 & 95.63 & 80.60 & 86.85 & 91.05 & 94.43 \\
\hline & 200 & 85.98 & 91.66 & 94.73 & 97.18 & 84.84 & 91.10 & 94.34 & 96.79 & 82.57 & 88.73 & 93.07 & 96.19 \\
\hline & 400 & 87.71 & 93.14 & 96.11 & 98.02 & 86.90 & 92.54 & 95.72 & 97.70 & 85.12 & 91.48 & 94.84 & 97.09 \\
\hline & 800 & 89.12 & 94.72 & 97.06 & 98.69 & 88.57 & 93.99 & 96.59 & 98.32 & 86.75 & 92.39 & 95.54 & 97.91 \\
\hline & 1600 & 89.27 & 94.25 & 97.10 & 98.70 & 88.43 & 93.79 & 96.48 & 98.47 & 87.15 & 92.52 & 95.82 & 98.07 \\
\hline & 3200 & 89.48 & 94.48 & 97.08 & 98.66 & 88.67 & 94.10 & 97.04 & 98.56 & 88.01 & 93.53 & 96.49 & 98.37 \\
\hline & & & & & & La & place & nnovat & ions & & & & \\
\hline PR-bw & 50 & 83.25 & 88.94 & 92.39 & 95.36 & 80.64 & 87.05 & 90.77 & 94.10 & 74.72 & 81.98 & 86.77 & 91.09 \\
\hline & 100 & 86.51 & 92.03 & 94.97 & 97.47 & 83.73 & 89.82 & 93.46 & 96.23 & 77.85 & 85.15 & 89.54 & 93.49 \\
\hline & 200 & 87.77 & 93.53 & 96.41 & 98.57 & 85.32 & 91.19 & 94.78 & 97.58 & 79.49 & 86.72 & 91.17 & 94.62 \\
\hline & 400 & 88.77 & 93.98 & 96.80 & 98.56 & 85.31 & 91.91 & 95.33 & 97.73 & 79.86 & 87.18 & 91.70 & 95.42 \\
\hline & 800 & 89.04 & 94.21 & 96.77 & 98.55 & 86.41 & 92.25 & 95.47 & 97.59 & 81.61 & 88.69 & 92.80 & 96.02 \\
\hline & 1600 & 88.94 & 94.36 & 97.19 & 98.63 & 86.48 & 92.63 & 96.08 & 98.03 & 82.76 & 89.81 & 93.76 & 96.61 \\
\hline & 3200 & 89.75 & 94.67 & 97.20 & 98.68 & 87.58 & 93.17 & 96.14 & 98.22 & 84.26 & 90.81 & 94.31 & 97.08 \\
\hline NW-bw & 50 & 82.69 & 88.51 & 92.11 & 94.94 & 80.65 & 86.92 & 90.76 & 93.57 & 75.48 & 82.51 & 87.23 & 91.21 \\
\hline & 100 & 86.71 & 92.14 & 94.76 & 97.16 & 84.06 & 90.11 & 93.37 & 96.28 & 78.68 & 85.60 & 90.07 & 93.84 \\
\hline & 200 & 88.06 & 93.55 & 96.52 & 98.65 & 85.49 & 91.35 & 94.96 & 97.75 & 80.09 & 87.04 & 91.34 & 94.90 \\
\hline & 400 & 88.84 & 94.00 & 96.80 & 98.60 & 85.50 & 92.05 & 95.33 & 97.81 & 80.13 & 87.30 & 91.83 & 95.50 \\
\hline & 800 & 89.15 & 94.21 & 96.74 & 98.45 & 86.46 & 92.26 & 95.50 & 97.65 & 81.60 & 88.69 & 92.80 & 95.91 \\
\hline & 1600 & 88.93 & 94.35 & 97.17 & 98.66 & 86.46 & 92.65 & 96.07 & 98.05 & 82.71 & 89.72 & 93.70 & 96.65 \\
\hline & 3200 & 89.82 & 94.68 & 97.18 & 98.65 & 87.57 & 93.14 & 96.10 & 98.22 & 84.07 & 90.62 & 94.27 & 97.06 \\
\hline naive & 50 & 77.79 & 84.28 & 88.78 & 92.46 & 75.40 & 82.15 & 86.65 & 90.50 & 70.25 & 77.66 & 82.79 & 87.17 \\
\hline & 100 & 82.76 & 88.58 & 92.31 & 95.24 & 80.00 & 86.34 & 90.55 & 93.94 & 75.42 & 82.50 & 87.31 & 91.04 \\
\hline & 200 & 85.29 & 91.11 & 94.52 & 97.10 & 83.23 & 89.17 & 92.89 & 95.95 & 79.16 & 85.37 & 90.00 & 93.74 \\
\hline & 400 & 86.95 & 92.42 & 95.78 & 97.89 & 84.52 & 91.03 & 94.61 & 97.15 & 80.83 & 87.78 & 91.94 & 95.22 \\
\hline & 800 & 88.27 & 93.41 & 96.16 & 98.03 & 86.14 & 91.92 & 95.05 & 97.19 & 83.32 & 89.75 & 93.49 & 96.25 \\
\hline & 1600 & 88.28 & 93.83 & 96.89 & 98.51 & 86.67 & 92.95 & 96.00 & 98.12 & 84.64 & 91.23 & 94.71 & 97.19 \\
\hline & 3200 & 89.52 & 94.39 & 96.93 & 98.63 & 88.19 & 93.51 & 96.35 & 98.29 & 86.21 & 91.91 & 95.07 & 97.67 \\
\hline & & & & & & Stud & 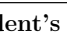 & $T$ & tions & & & & \\
\hline PR-bw & 50 & 83.99 & 89.70 & 93.44 & 95.72 & 81.32 & 87.78 & 91.76 & 94.57 & 74.80 & 81.94 & 87.09 & 91.01 \\
\hline & 100 & 86.72 & 92.08 & 95.43 & 97.57 & 84.08 & 90.09 & 93.82 & 96.49 & 77.32 & 84.91 & 89.39 & 93.31 \\
\hline & 200 & 87.91 & 93.00 & 96.20 & 98.20 & 84.87 & 90.97 & 94.60 & 97.14 & 78.38 & 85.80 & 90.32 & 94.22 \\
\hline & 400 & 88.73 & 93.92 & 96.64 & 98.45 & 85.19 & 91.41 & 94.83 & 97.34 & 78.40 & 86.22 & 90.68 & 94.51 \\
\hline & 800 & 88.99 & 94.18 & 96.84 & 98.52 & 85.74 & 91.68 & 95.00 & 97.37 & 80.18 & 87.43 & 91.65 & 94.97 \\
\hline & 1600 & 89.36 & 94.39 & 97.06 & 98.74 & 86.25 & 92.12 & 95.49 & 98.02 & 81.33 & 88.30 & 92.46 & 96.03 \\
\hline & 3200 & 89.60 & 94.62 & 97.17 & 98.81 & 86.96 & 92.78 & 95.85 & 97.91 & 83.16 & 89.89 & 93.66 & 96.52 \\
\hline NW-bw & 50 & 83.62 & 89.16 & 92.37 & 95.22 & 81.04 & 87.53 & 91.45 & 94.23 & 75.47 & 82.21 & 87.16 & 91.06 \\
\hline & 100 & 86.54 & 92.00 & 95.18 & 97.43 & 84.18 & 90.45 & 93.90 & 96.37 & 77.70 & 85.41 & 89.83 & 93.48 \\
\hline & 200 & 88.14 & 92.94 & 96.29 & 98.20 & 85.24 & 91.08 & 94.75 & 97.32 & 78.61 & 86.22 & 90.76 & 94.35 \\
\hline & 400 & 88.65 & 93.93 & 96.74 & 98.53 & 85.26 & 91.59 & 94.98 & 97.41 & 78.72 & 86.32 & 90.76 & 94.76 \\
\hline & 800 & 89.01 & 94.11 & 96.94 & 98.55 & 85.63 & 91.68 & 95.10 & 97.40 & 80.26 & 87.43 & 91.67 & 95.06 \\
\hline & 1600 & 89.31 & 94.38 & 97.11 & 98.80 & 86.20 & 92.13 & 95.52 & 98.00 & 81.22 & 88.22 & 92.40 & 96.03 \\
\hline & 3200 & 89.68 & 94.60 & 97.17 & 98.85 & 86.84 & 92.78 & 95.83 & 97.91 & 82.94 & 89.76 & 93.55 & 96.48 \\
\hline naive & 50 & 78.70 & 85.36 & 89.29 & 92.75 & 75.92 & 83.14 & 87.52 & 91.45 & 70.33 & 77.74 & 82.78 & 87.71 \\
\hline & 100 & 82.37 & 88.68 & 92.57 & 95.41 & 79.60 & 86.44 & 90.68 & 93.98 & 74.09 & 81.61 & 86.65 & 90.69 \\
\hline & 200 & 85.18 & 91.01 & 94.33 & 96.95 & 82.53 & 88.90 & 92.83 & 95.88 & 77.52 & 84.62 & 89.69 & 93.21 \\
\hline & 400 & 87.06 & 92.53 & 95.78 & 97.88 & 84.01 & 90.34 & 94.02 & 96.68 & 79.68 & 86.44 & 90.75 & 94.28 \\
\hline & 800 & 87.92 & 93.19 & 96.15 & 98.11 & 85.45 & 91.42 & 94.83 & 97.27 & 81.87 & 88.58 & 92.50 & 95.40 \\
\hline & 1600 & 88.91 & 93.87 & 96.71 & 98.53 & 86.49 & 92.45 & 95.62 & 98.12 & 83.28 & 89.84 & 93.69 & 96.64 \\
\hline & 3200 & 89.32 & 94.45 & 96.93 & 98.76 & 87.70 & 93.04 & 96.12 & 98.15 & 85.26 & 91.44 & 94.51 & 96.98 \\
\hline
\end{tabular}

Table 4: Simulated percentage coverages of the CIs for the Sharpe Ratio in the general noti.i.d. setting. 


\begin{tabular}{|c|c|c|c|c|c|c|c|c|c|c|c|c|c|}
\hline \multirow{2}{*}{\multicolumn{2}{|c|}{$\begin{array}{c}\text { Value of } v \\
100(1-\alpha) \%\end{array}$}} & \multicolumn{4}{|c|}{ small } & \multicolumn{4}{|c|}{ medium } & \multicolumn{4}{|c|}{ high } \\
\hline & & \multirow[t]{2}{*}{$\overline{90 \%}$} & \multirow[t]{2}{*}{$95 \%$} & \multirow[t]{2}{*}{$97.5 \%$} & \multirow[t]{2}{*}{$99 \%$} & $90 \%$ & $95 \%$ & $97.5 \%$ & $99 \%$ & $\overline{90 \%}$ & $95 \%$ & $97.5 \%$ & $99 \%$ \\
\hline bw & n & & & & & Gaı & ussian & Innova & tions & & & & \\
\hline PR-bw & 50 & 82.81 & 88.64 & 92.00 & 94.57 & 81.47 & 87.97 & 91.52 & 94.36 & 78.97 & 85.77 & 89.97 & 93.11 \\
\hline & 100 & 86.89 & 92.30 & 95.12 & 96.92 & 85.92 & 91.55 & 94.76 & 96.62 & 82.68 & 89.55 & 93.23 & 95.87 \\
\hline & 200 & 88.75 & 93.62 & 96.17 & 97.96 & 87.62 & 92.96 & 95.74 & 97.77 & 84.21 & 90.60 & 94.62 & 97.02 \\
\hline & 400 & 89.82 & 94.65 & 97.08 & 98.71 & 88.52 & 93.83 & 96.46 & 98.31 & 85.67 & 91.78 & 95.03 & 97.37 \\
\hline & 800 & 90.56 & 95.56 & 97.52 & 98.92 & 89.43 & 94.48 & 97.15 & 98.56 & 86.21 & 92.47 & 95.83 & 97.88 \\
\hline & 1600 & 89.78 & 94.73 & 97.32 & 98.87 & 88.97 & 94.12 & 96.77 & 98.60 & 86.43 & 92.28 & 95.56 & 97.79 \\
\hline & 3200 & 89.58 & 94.69 & 97.27 & 98.82 & 88.65 & 94.26 & 96.89 & 98.60 & 86.49 & 92.73 & 96.01 & 98.08 \\
\hline NW-bw & 50 & 81.93 & 87.60 & 91.37 & 94.12 & 82.79 & 88.34 & 91.69 & 94.32 & 85.62 & 89.98 & 92.88 & 94.90 \\
\hline & 100 & 85.06 & 90.59 & 93.57 & 96.01 & 85.24 & 90.76 & 93.64 & 96.08 & 85.53 & 90.67 & 93.68 & 95.83 \\
\hline & 200 & 87.16 & 92.38 & 95.43 & 97.31 & 86.77 & 92.20 & 95.16 & 97.27 & 85.55 & 91.47 & 94.77 & 97.03 \\
\hline & 400 & 89.59 & 94.45 & 96.84 & 98.50 & 88.49 & 93.91 & 96.33 & 98.05 & 86.31 & 92.17 & 95.45 & 97.47 \\
\hline & 800 & 90.54 & 95.48 & 97.48 & 98.93 & 89.54 & 94.55 & 97.14 & 98.55 & 86.80 & 93.15 & 95.88 & 97.96 \\
\hline & 1600 & 89.84 & 94.78 & 97.33 & 98.96 & 89.01 & 94.23 & 96.88 & 98.66 & 86.82 & 92.50 & 95.61 & 98.03 \\
\hline & 3200 & 89.68 & 94.73 & 97.25 & 98.81 & 88.76 & 94.30 & 97.04 & 98.62 & 86.73 & 92.77 & 96.12 & 98.13 \\
\hline naive & 50 & 79.61 & 86.34 & 90.39 & 93.66 & 78.75 & 85.64 & 90.22 & 93.66 & 76.38 & 83.84 & 88.82 & 92.69 \\
\hline & 100 & 83.92 & 89.91 & 93.28 & 96.10 & 83.10 & 89.19 & 93.08 & 95.75 & 80.33 & 87.51 & 91.53 & 94.71 \\
\hline & 200 & 86.10 & 91.61 & 94.63 & 97.00 & 85.14 & 91.06 & 94.51 & 96.92 & 82.72 & 89.38 & 93.29 & 96.40 \\
\hline & 400 & 87.87 & 93.13 & 95.86 & 97.90 & 87.08 & 92.54 & 95.55 & 97.57 & 84.87 & 90.91 & 94.51 & 96.83 \\
\hline & 800 & 89.41 & 94.73 & 97.00 & 98.66 & 88.69 & 93.93 & 96.61 & 98.32 & 86.81 & 92.47 & 95.68 & 97.96 \\
\hline & 1600 & 89.34 & 94.33 & 96.96 & 98.58 & 88.43 & 93.67 & 96.56 & 98.40 & 87.11 & 92.68 & 95.68 & 98.06 \\
\hline & 3200 & 89.44 & 94.52 & 97.14 & 98.70 & 88.62 & 94.16 & 96.90 & 98.57 & 87.53 & 93.50 & 96.51 & 98.30 \\
\hline & & & & & & La & place & inova & ions & & & & \\
\hline PR-bw & 50 & 82.57 & 88.13 & 91.63 & 94.17 & 80.53 & 87.09 & 91.09 & 93.97 & 75.97 & 83.57 & 88.34 & 92.07 \\
\hline & 100 & 86.96 & 92.04 & 94.84 & 96.66 & 84.65 & 90.46 & 93.80 & 96.19 & 80.24 & 87.11 & 91.37 & 94.63 \\
\hline & 200 & 88.62 & 93.97 & 96.64 & 98.23 & 86.17 & 91.98 & 95.47 & 97.60 & 81.45 & 88.45 & 92.57 & 95.95 \\
\hline & 400 & 89.32 & 94.41 & 97.02 & 98.62 & 86.66 & 92.70 & 95.87 & 98.11 & 81.57 & 88.89 & 93.36 & 96.60 \\
\hline & 800 & 89.32 & 94.28 & 96.93 & 98.52 & 86.90 & 92.61 & 95.76 & 97.97 & 82.91 & 89.67 & 93.54 & 96.62 \\
\hline & 1600 & 89.17 & 94.54 & 97.16 & 98.72 & 86.89 & 92.87 & 96.09 & 98.23 & 83.28 & 89.88 & 94.05 & 96.91 \\
\hline & 3200 & 89.94 & 94.77 & 97.15 & 98.74 & 87.80 & 93.22 & 96.18 & 98.35 & 84.68 & 90.85 & 94.81 & 97.32 \\
\hline NW-bw & 50 & 81.94 & 88.01 & 91.28 & 93.94 & 82.84 & 88.16 & 91.38 & 94.11 & 83.76 & 88.59 & 91.46 & 94.03 \\
\hline & 100 & 85.41 & 90.50 & 93.70 & 95.92 & 84.55 & 90.01 & 93.23 & 95.81 & 83.48 & 89.43 & 92.66 & 95.23 \\
\hline & 200 & 87.73 & 93.20 & 95.85 & 97.68 & 85.72 & 91.61 & 95.13 & 97.34 & 82.78 & 89.15 & 93.14 & 96.21 \\
\hline & 400 & 88.95 & 94.03 & 96.77 & 98.51 & 86.55 & 92.65 & 95.57 & 98.12 & 82.21 & 89.08 & 93.65 & 96.63 \\
\hline & 800 & 89.31 & 94.25 & 96.80 & 98.49 & 87.17 & 92.83 & 95.86 & 97.95 & 83.48 & 90.02 & 93.78 & 96.68 \\
\hline & 1600 & 89.30 & 94.60 & 97.16 & 98.76 & 87.19 & 92.93 & 96.17 & 98.27 & 83.66 & 90.11 & 94.25 & 96.93 \\
\hline & 3200 & 90.00 & 94.80 & 97.17 & 98.78 & 88.02 & 93.36 & 96.26 & 98.42 & 84.76 & 91.11 & 94.91 & 97.29 \\
\hline naive & 50 & 78.93 & 85.27 & 89.39 & 92.68 & 77.39 & 84.04 & 88.60 & 92.57 & 72.73 & 81.01 & 86.43 & 90.89 \\
\hline & 100 & 83.27 & 89.37 & 92.93 & 95.58 & 81.40 & 87.97 & 92.10 & 95.20 & 77.54 & 85.13 & 89.71 & 93.46 \\
\hline & 200 & 85.82 & 91.75 & 94.78 & 97.18 & 83.61 & 89.95 & & 96.69 & 79.75 & 86.69 & 91.24 & 94.97 \\
\hline & 400 & 87.20 & 92.60 & 95.65 & 97.83 & 85.03 & 91.19 & 94.91 & 97.43 & 81.22 & 88.15 & 92.87 & 95.95 \\
\hline & 800 & 88.24 & 93.42 & 96.10 & 98.12 & 86.36 & 91.99 & 95.44 & 97.69 & 83.42 & 89.87 & 93.92 & 96.56 \\
\hline & 1600 & 88.53 & 94.02 & 96.76 & 98.51 & 86.93 & 92.77 & 96.03 & 98.14 & 84.42 & 91.02 & 94.83 & 97.10 \\
\hline & 3200 & 89.59 & 94.41 & 96.98 & 98.65 & 87.91 & 93.51 & 96.34 & 98.33 & 86.15 & 92.10 & 95.45 & 97.74 \\
\hline & & & & & & Stud & 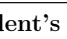 & & IIOns & & & & \\
\hline PR-bw & 50 & 82.98 & 88.48 & 91.60 & 94.25 & 80.46 & 86.89 & 90.60 & 93.80 & 75.08 & 82.76 & 87.55 & 91.38 \\
\hline & 100 & 86.56 & 91.98 & 94.54 & 96.68 & 83.76 & 90.05 & 93.69 & 96.19 & 77.84 & 85.43 & 90.38 & 94.35 \\
\hline & 200 & 88.11 & 93.44 & 96.02 & 98.12 & 85.37 & 91.39 & 94.65 & 97.34 & 78.79 & 86.66 & 91.44 & 95.20 \\
\hline & 400 & 89.08 & 94.45 & 96.91 & 98.52 & 85.86 & 91.66 & 95.10 & 97.58 & 79.09 & 86.89 & 91.39 & 95.23 \\
\hline & 800 & 88.96 & 94.09 & 96.76 & 98.60 & 85.56 & 92.00 & 95.08 & 97.54 & 80.16 & 87.27 & 91.59 & 95.06 \\
\hline & 1600 & 89.50 & 94.41 & 97.09 & 98.83 & 86.09 & 92.03 & 95.55 & 98.04 & 80.96 & 87.62 & 92.42 & 95.93 \\
\hline & 3200 & 89.74 & 94.67 & 97.17 & 98.73 & 86.84 & 92.89 & 95.87 & 97.98 & 82.44 & 89.09 & 93.21 & 96.30 \\
\hline NW-bw & 50 & 82.08 & 87.72 & 90.82 & 93.67 & 82.88 & 88.12 & 91.31 & 94.00 & 83.67 & 89.03 & 91.63 & 93.95 \\
\hline & 100 & 84.95 & 90.37 & 93.20 & 95.73 & 83.85 & 89.48 & 93.03 & 95.79 & 82.02 & 88.23 & 92.11 & 94.96 \\
\hline & 200 & 87.41 & 92.51 & 95.34 & 97.59 & 85.18 & 91.18 & 94.46 & 96.85 & 80.96 & 88.18 & 92.47 & 95.69 \\
\hline & 400 & 88.82 & 94.14 & 96.77 & 98.31 & 85.86 & 91.54 & 94.90 & 97.50 & 79.88 & 87.27 & 91.75 & 95.17 \\
\hline & 800 & 88.80 & 93.70 & 96.69 & 98.65 & 85.83 & 91.99 & 95.05 & 97.61 & 80.55 & 87.54 & 91.92 & 95.20 \\
\hline & 1600 & 89.54 & 94.48 & 97.11 & 98.82 & 86.37 & 92.18 & 95.66 & 98.13 & 81.29 & 87.88 & 92.65 & 95.93 \\
\hline & 3200 & 89.88 & 94.76 & 97.26 & 98.72 & 87.16 & 92.87 & 95.93 & 97.96 & 82.54 & 89.14 & 93.18 & 96.25 \\
\hline naive & 50 & 80.02 & 86.14 & 89.72 & 92.84 & 77.16 & 84.45 & 89.20 & 92.75 & 71.65 & 80.24 & 85.87 & 90.64 \\
\hline & 100 & 83.01 & 89.10 & 92.54 & 95.21 & 80.38 & 87.08 & 91.50 & 94.83 & 74.92 & 82.59 & 87.77 & 92.56 \\
\hline & 200 & 85.65 & 90.88 & 94.35 & 96.79 & 82.84 & 89.24 & 93.11 & 96.19 & 77.24 & 85.10 & 89.98 & 93.83 \\
\hline & 400 & 87.22 & 92.85 & 95.75 & 97.79 & 84.37 & 90.53 & 94.27 & 96.73 & 78.91 & 86.14 & 91.03 & 94.55 \\
\hline & 800 & 88.03 & 93.00 & 95.95 & 98.05 & 84.56 & 91.17 & 94.51 & 97.26 & 80.74 & 87.71 & 91.80 & 95.14 \\
\hline & 1600 & 88.94 & 93.91 & 96.70 & 98.60 & 86.24 & 92.07 & 95.45 & 98.10 & 82.37 & 88.60 & 93.12 & 96.29 \\
\hline & 3200 & 89.39 & 94.51 & 96.91 & 98.62 & 87.28 & 93.09 & 96.02 & 98.26 & 84.16 & 90.42 & 94.13 & 96.89 \\
\hline
\end{tabular}

Table 5: Simulated percentage coverages of the CIs for the Sortino Ratio in the general not-i.i.d. setting. 


\begin{tabular}{|c|c|c|c|c|c|c|c|c|c|c|c|c|c|}
\hline \multirow{2}{*}{\multicolumn{2}{|c|}{$\begin{array}{c}\text { Value of } \omega \\
100(1-\alpha) \%\end{array}$}} & \multicolumn{4}{|c|}{ small } & \multicolumn{4}{|c|}{ medium } & \multicolumn{4}{|c|}{ high } \\
\hline & & \multirow[t]{2}{*}{$\overline{90 \%}$} & \multirow[t]{2}{*}{$95 \%$} & \multirow[t]{2}{*}{$97.5 \%$} & \multirow[t]{2}{*}{$99 \%$} & $90 \%$ & $95 \%$ & $97.5 \%$ & $99 \%$ & $\overline{90 \%}$ & $95 \%$ & $97.5 \%$ & $99 \%$ \\
\hline bw & n & & & & & & & Innova & tions & & & & \\
\hline PR-bw & 50 & 81.40 & 86.69 & 89.95 & 92.89 & 81.16 & 86.84 & 90.09 & 92.95 & 79.99 & 85.73 & 88.96 & 91.97 \\
\hline & 100 & 86.08 & 91.04 & 93.46 & 95.61 & 85.90 & 90.38 & 93.18 & 95.50 & 83.74 & 89.50 & 92.36 & 94.39 \\
\hline & 200 & 88.27 & 92.99 & 95.62 & 97.41 & 87.10 & 92.46 & 95.37 & 97.10 & 84.98 & 90.72 & 94.08 & 96.35 \\
\hline & 400 & 89.92 & 94.44 & 96.81 & 98.49 & 88.84 & 93.73 & 96.38 & 98.14 & 86.32 & 92.23 & 95.27 & 97.40 \\
\hline & 800 & 90.72 & 95.44 & 97.52 & 98.90 & 89.70 & 94.81 & 97.07 & 98.52 & 86.90 & 92.75 & 95.90 & 98.10 \\
\hline & 1600 & 89.95 & 94.82 & 97.34 & 98.85 & 88.82 & 94.15 & 96.83 & 98.66 & 86.62 & 92.55 & 95.80 & 97.87 \\
\hline & 3200 & 89.73 & 94.70 & 97.22 & 98.79 & 88.98 & 94.39 & 96.94 & 98.62 & 87.29 & 93.08 & 96.07 & 98.10 \\
\hline NW-bw & 50 & 84.26 & 88.78 & 91.42 & 93.60 & 83.54 & 88.28 & 91.19 & 93.59 & 81.46 & 86.70 & 89.85 & 92.61 \\
\hline & 100 & 87.62 & 91.94 & 94.30 & 96.11 & 86.70 & 91.17 & 93.46 & 95.58 & 84.10 & 89.72 & 92.62 & 94.72 \\
\hline & 200 & 88.68 & 93.06 & 95.67 & 97.38 & 87.45 & 92.49 & 95.25 & 97.17 & 85.42 & 91.16 & 94.20 & 96.41 \\
\hline & 400 & 90.26 & 94.54 & 96.97 & 98.39 & 88.93 & 93.92 & 96.50 & 98.15 & 86.54 & 92.47 & 95.40 & 97.55 \\
\hline & 800 & 90.84 & 95.41 & 97.47 & 98.87 & 89.97 & 94.87 & 97.14 & 98.60 & 87.22 & 92.97 & 96.11 & 98.13 \\
\hline & 1600 & 90.06 & 94.94 & 97.24 & 98.86 & 88.97 & 94.32 & 96.89 & 98.65 & 86.78 & 92.68 & 95.78 & 97.91 \\
\hline & 3200 & 89.79 & 94.78 & 97.27 & 98.80 & 89.02 & 94.57 & 96.99 & 98.62 & 87.22 & 93.12 & 96.06 & 98.10 \\
\hline naive & 50 & 80.12 & 85.98 & 89.74 & 92.75 & 80.17 & 86.38 & 90.04 & 92.97 & 79.44 & 85.75 & 89.68 & 92.50 \\
\hline & 100 & 84.14 & 89.78 & 92.92 & 95.26 & 83.89 & 89.64 & 92.96 & 95.24 & 82.66 & 88.84 & 92.35 & 94.65 \\
\hline & 200 & 86.00 & 91.14 & 94.44 & 96.63 & 85.44 & 91.12 & 94.27 & 96.50 & 83.85 & 90.16 & 93.35 & 95.98 \\
\hline & 400 & 87.91 & 93.10 & 95.81 & 97.77 & 87.40 & 92.57 & 95.49 & 97.59 & 86.00 & 91.65 & 94.73 & 97.02 \\
\hline & 800 & 89.51 & 94.72 & 96.88 & 98.51 & 88.87 & 94.19 & 96.73 & 98.23 & 87.36 & 93.06 & 95.89 & 98.05 \\
\hline & 1600 & 89.29 & 94.33 & 96.97 & 98.49 & 88.54 & 93.84 & 96.65 & 98.40 & 87.52 & 92.98 & 96.13 & 98.09 \\
\hline & 3200 & 89.49 & 94.46 & 97.07 & 98.66 & 88.95 & 94.31 & 96.95 & 98.59 & 88.27 & 93.81 & 96.59 & 98.29 \\
\hline & & & & & & La & place & anova & ions & & & & \\
\hline PR-bw & 50 & 81.44 & 86.40 & 89.35 & 92.20 & 81.21 & 86.46 & 89.80 & 92.46 & 80.66 & 85.47 & 88.79 & 91.42 \\
\hline & 100 & 86.21 & 91.04 & 93.80 & 95.77 & 85.34 & 90.78 & 93.47 & 95.30 & 83.07 & 88.92 & 91.71 & 94.32 \\
\hline & 200 & 88.21 & 93.19 & 95.86 & 97.54 & 86.82 & 92.57 & 95.41 & 97.25 & 84.40 & 90.60 & 93.64 & 96.27 \\
\hline & 400 & 89.33 & 94.31 & 96.70 & 98.35 & 88.05 & 93.22 & 96.48 & 98.16 & 84.95 & 91.34 & 94.96 & 97.18 \\
\hline & 800 & 89.42 & 94.30 & 96.75 & 98.52 & 87.62 & 93.18 & 96.28 & 98.29 & 84.85 & 91.05 & 94.81 & 97.24 \\
\hline & 1600 & 89.33 & 94.60 & 97.11 & 98.75 & 87.90 & 93.61 & 96.55 & 98.40 & 85.09 & 91.69 & 95.28 & 97.70 \\
\hline & 3200 & 89.98 & 94.96 & 97.24 & 98.81 & 88.84 & 93.81 & 96.57 & 98.55 & 86.45 & 92.16 & 95.62 & 97.97 \\
\hline NW-bw & 50 & 84.64 & 89.16 & 91.51 & 93.74 & 83.64 & 88.40 & 91.31 & 93.43 & 81.85 & 86.66 & 89.62 & 92.21 \\
\hline & 100 & 87.65 & 92.25 & 94.66 & 96.41 & 86.39 & 91.52 & 93.96 & 95.97 & 84.12 & 89.38 & 92.47 & 94.68 \\
\hline & 200 & 89.17 & 94.07 & 96.22 & 97.77 & 87.30 & 93.05 & & 97.39 & 85.19 & 91.08 & 94.04 & 96.49 \\
\hline & 400 & 89.56 & 94.35 & 96.94 & 98.39 & 88.34 & 93.44 & 96.51 & 98.22 & 85.48 & 91.59 & 95.15 & 97.34 \\
\hline & 800 & 89.61 & 94.42 & 96.72 & 98.46 & 87.75 & 93.42 & 96.35 & 98.33 & 85.21 & 91.26 & 94.97 & 97.30 \\
\hline & 1600 & 89.42 & 94.71 & 97.14 & 98.79 & 88.08 & 93.72 & 96.69 & 98.44 & 85.19 & 91.70 & 95.39 & 97.79 \\
\hline & 3200 & 90.01 & 95.05 & 97.33 & 98.81 & 88.85 & 93.91 & 96.64 & 98.56 & 86.33 & 92.01 & 95.56 & 97.93 \\
\hline naive & 50 & 79.18 & 84.96 & 88.52 & 91.65 & 79.18 & 85.67 & 89.32 & 92.25 & 79.53 & 85.76 & 89.27 & 91.55 \\
\hline & 100 & 83.59 & 89.12 & 92.34 & 94.92 & 83.07 & 89.22 & 92.69 & 95.37 & 81.98 & 88.06 & 91.87 & 94.60 \\
\hline & 200 & 85.90 & 91.60 & 94.63 & 96.70 & 84.85 & 91.09 & & 96.70 & 83.20 & 89.80 & 93.52 & 95.88 \\
\hline & 400 & 87.36 & 92.56 & 95.56 & 97.63 & 86.78 & 92.24 & 95.44 & 97.77 & 84.63 & 91.16 & 94.63 & 97.23 \\
\hline & 800 & 88.38 & 93.41 & 95.96 & 97.99 & 87.17 & 92.67 & 95.82 & 97.97 & 85.74 & 91.59 & 95.13 & 97.45 \\
\hline & 1600 & 88.48 & 94.03 & 96.73 & 98.56 & 87.94 & 93.47 & 96.57 & 98.30 & 86.75 & 92.47 & 95.95 & 97.95 \\
\hline & 3200 & 89.61 & 94.60 & 97.10 & 98.72 & 88.98 & 94.02 & 96.79 & 98.54 & 87.96 & 93.31 & 96.39 & 98.28 \\
\hline & & & & & & Stud & 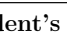 & & tions & & & & \\
\hline PR-bw & 50 & 81.69 & 86.79 & & 92.21 & 80.92 & 86.42 & 89.75 & 92.37 & 79.45 & 85.01 & 88.19 & 91.28 \\
\hline & 100 & 85.74 & 90.41 & 93.18 & 95.46 & 84.55 & 89.96 & 93.26 & 95.35 & 82.09 & 88.33 & 91.67 & 94.19 \\
\hline & 200 & 87.98 & 93.05 & 95.72 & 97.59 & 86.75 & 92.11 & 94.96 & 97.11 & 83.70 & 90.10 & 93.56 & 95.99 \\
\hline & 400 & 89.17 & 94.40 & 96.94 & 98.29 & 87.76 & 93.11 & 96.23 & 97.94 & 84.17 & 90.82 & 94.44 & 96.73 \\
\hline & 800 & 89.14 & 94.16 & 96.72 & 98.52 & 87.40 & 93.06 & 96.01 & 97.91 & 84.38 & 90.64 & 94.38 & 96.98 \\
\hline & 1600 & 89.66 & 94.66 & 97.33 & 98.86 & 88.27 & 93.76 & 96.64 & 98.52 & 85.19 & 91.37 & 95.21 & 97.56 \\
\hline & 3200 & 90.03 & 94.80 & 97.30 & 98.78 & 88.67 & 93.94 & 96.77 & 98.50 & 86.38 & 92.18 & 95.39 & 97.79 \\
\hline NW-bw & 50 & 84.97 & 89.21 & 91.85 & 93.86 & 83.54 & 88.32 & 91.52 & 93.75 & 80.99 & 86.48 & 89.51 & 92.08 \\
\hline & 100 & 87.73 & 91.82 & 94.05 & 95.85 & 85.83 & 90.71 & 93.54 & 95.67 & 82.84 & 88.94 & 92.22 & 94.61 \\
\hline & 200 & 89.00 & 93.72 & 96.10 & 97.66 & 87.45 & 92.66 & 95.16 & 97.11 & 84.40 & 90.56 & 94.09 & 96.17 \\
\hline & 400 & 89.62 & 94.48 & 97.02 & 98.38 & 88.17 & 93.27 & 96.41 & 98.06 & 85.00 & 91.29 & 94.79 & 97.02 \\
\hline & 800 & 89.20 & 94.13 & 96.83 & 98.56 & 87.64 & 93.33 & 96.15 & 98.00 & 84.72 & 90.90 & 94.68 & 97.09 \\
\hline & 1600 & 89.65 & 94.85 & 97.32 & 98.88 & 88.45 & 93.82 & 96.70 & 98.63 & 85.41 & 91.37 & 95.27 & 97.58 \\
\hline & 3200 & 90.24 & 94.92 & 97.35 & 98.82 & 88.77 & 94.05 & 96.75 & 98.58 & 86.27 & 92.16 & 95.34 & 97.71 \\
\hline naive & 50 & 80.10 & 85.75 & 89.30 & 92.09 & 79.71 & 85.60 & 89.40 & 92.47 & 78.59 & 84.95 & 88.86 & 91.81 \\
\hline & 100 & 83.35 & 88.92 & 92.15 & 94.66 & 82.37 & 88.77 & 92.07 & 94.81 & 80.50 & 87.25 & 91.30 & 94.14 \\
\hline & 200 & 86.00 & 90.97 & 94.04 & 96.45 & 84.80 & 90.57 & 93.88 & 96.27 & 82.80 & 89.44 & 93.00 & 95.75 \\
\hline & 400 & 87.30 & 93.08 & 95.86 & 97.68 & 86.24 & 92.38 & 95.48 & 97.56 & 84.24 & 90.76 & 94.33 & 96.79 \\
\hline & 800 & 88.15 & 93.01 & 95.96 & 98.07 & 86.99 & 92.64 & 95.72 & 97.76 & 85.25 & 91.17 & 94.87 & 97.08 \\
\hline & 1600 & 89.10 & 94.08 & 96.86 & 98.59 & 88.32 & 93.74 & 96.71 & 98.45 & 86.68 & 92.29 & 95.82 & 98.12 \\
\hline & 3200 & 89.54 & 94.57 & 97.15 & 98.68 & 89.08 & 94.06 & 96.79 & 98.54 & 88.00 & 93.22 & 96.23 & 98.20 \\
\hline
\end{tabular}

Table 6: Simulated percentage coverages of the CIs for the Omega Ratio in the general noti.i.d. setting. 
returns. This means that about 15 years of daily observations are not sufficient in order have, in general, a good coverage accuracy. We emphasize that, in this context, a good interval estimator for the performance ratios would be very useful since the estimators of this ratio have a huge variability. This fact is highlighted by the expressions of the variance of the estimators and by the simulations results which, for brevity, are not given here. The high variability of these estimators is due to the fact that they are defined as the ratio of two random variables. Indeed, it is well known that a "ratio random variable" has usually a high variability. For example, the ratio of two independent normal random variables follows the Chauchy distribution which do not possesses moments; a similar example is given by the ratio of two independent uniform random variables; others examples are the $F$ and Student's $t$ distributions which do not posses the moments of all order even if they are defined as the ratio of two random variables whit all moments.

Concluding, we think that the negative result obtained by the simulation study suggests that the performance ratios considered should be applied with caution especially when they are used to address the investment choices.

\section{References}

Andrews, D.W.K. (1991), Heteroscedasticity and autocorrelation consistent covariance matrix estimation, Econometrica, 59, 81758.

Andrews, D.W.K. and J.C. Monahan (1992), An improved heteroskedasticity and autocorrelation consistent covariance matrix estimator. Econometrica,60, 953-966.

Billingsley P. (1995), Probability and Measure, 3rd ed, Wiley.

Billingsley P. (1999), Convergence of Probability Measure, 2nd ed., Wiley.

Brockwell P.J. and R.A. Davis (1991), Time Series: Theory and Methods, 2nd ed, Springer Verlag.

Carrasco, M. and X. Chen (2002), Mixing and moment properties of various GARCH and stochastic volatility models, Econometric Theory, 18, 1739.

Davidson J. (1994), Stochastic Limit Theory: An Introduction for Econometricians, Oxford University Press.

De Capitani, L. and M. Zenga (2011). Point and interval estimation for some financial performance measures. forthcoming on Statistica 63 Applicazioni.

De Capitani, L. (2010), Interval Estimation for the Sharpe Ratio when returns are not i.i.d. with special emphasis on the $G A R C H(1,1)$ process with symmetric innovations, Quaderni del dipartimento dei Metodi Qantitativi per le Scienze economiche ed Aziendali, Univerist degli Studi di Milano-Bicocca.

Farinelli, S., M. Ferreira, D. Rossello, M. Thoeny and L. Tibiletti (2008), Beyond Sharpe ratio: Optimal asset allocation using different performance ratios, Journal of Banking $\&$ Finance, 32, 2057-2063. 
Genay, R., M. Dacorogna, U. Muller, O. Picket and R. Olsen (2001), An introduction to high-frequency finance, Academic Press, San Diego.

Hall A.R. (2005), Generalized Method of Moments, Oxford University Press.

Hall P. and C.C. Heyde (1980), Martingale Limit Theory and Its Application, Academic Press.

Ibragimov I.A. (1962), Some limit theorems for stationary processes, Theory of Probability and its Applications, 7, 349-82.

Karlin S. and H.M. Taylor (1975), A first course in stochastic processes, Second edition, Academic Press, New York.

Keating, C. and W.F. Shadwick (2002), A Universal Performance Measure, Journal of Performance Measurement, 6, 59-84.

Konno, H. and H. Yamazaki, H. (1991), Mean-absolute deviation portfolio optimization model and its application to Tokio stock market, Management Science, 37, 519-531.

Lo, A.W. (2002), The Statistics of Sharpe Ratio, Financial Analyst Journal, 58, 36-52.

Newey W.K. and K.D. West (1987), A Simple, Positive Semi-Definite, Heteroskedasticity and Autocorrelation Consistent Covariance Matrix, Econometrica, 55, 703-708.

Newey W.K. and K.D. West (1994), Automatic lag selection in covariance matrix estimation, Review of Economic Studies, 61, 631-653.

Opdike J.D. (2007), Comparing Sharpe Ratios: So Where are the p-values?, Journal of Asset Management, 8 .

Serfling, R.J. (1980), Approximation Theorems of Mathematical Statistics, Wiley.

Shalit, H. and S. Yitzhaki (1984), Mean-Gini, Portfolio theory, and the pricing of risky asset, Journal of Finance, 39, 1449-1468.

Sharpe, W.F. (1964), Capital Asset Prices: A Theory of Market Equilibrium under Conditions of Risk, The Journal of Finance, 19, 425-442.

Sharpe, W.F. (1966), Mutual fund Performance, Journal of Business, January, 39, 119-138.

Sharpe, W.F. (1994), The Sharpe Ratio, Journal of Portfolio Management, 21, 49-58.

Sortino, F. and R. Van der Meer (1991), Downside risk, The Journal of Portfolio Management, $27,31$.

Sortino, F. and L. Price (1994), Performance measurement in a downside risk framenwork, The Journal of Investing, 59, 65. 